ESAIM: PROCEEDINGS, September 2005, Vol.14, 124-155

Eric Cancès \& Jean-Frédéric Gerbeau, Editors

DOI: $10.1051 /$ proc:2005011

\title{
DEVELOPMENT AND CALIBRATION OF A MODELING TOOL FOR THE ANALYSIS OF CLINICAL DATA IN HUMAN NUTRITION
}

\author{
Barbara Juillet ${ }^{1}$, Julien Salomon ${ }^{2}$, Daniel Tomé $^{1}$ and Hélène Fouillet ${ }^{1}$
}

\begin{abstract}
This paper addresses the problem of calibrating a compartmental model which describes the postprandial distribution of dietary nitrogen in humans after the ingestion of a protein meal. This type of problem (i.e., a classic inverse problem) requires optimization of an objective function that measures the goodness-of-fit of the model predictions to a given set of experimental data. In our particular case, traditional local, gradient-based optimization methods have failed to arrive at satisfactory solutions of the inverse problem because of the large number of parameters to be estimated, the high non-linearity of the objective function and the few experimental data accessible in humans. To overcome these limitations, we have developed a calibration method that uses all available information on the system behavior so as to divide the large inverse problem into many smaller sub-problems, on which a variant of the Nelder-Mead (NM) simplex search procedure was proven to be successful. This calibration method makes it possible to obtain solutions that are close to the optimal values of most of the model parameters, even when noisy experimental data are introduced in the objective function. Using these estimated parameters, it is now possible to correctly simulate the temporal evolution of all compartments of the physiological model, which constitutes a useful, explanatory tool to describe the different dynamic processes involved in the metabolic utilization of dietary proteins in humans.
\end{abstract}

Résumé. Nous abordons ici le problème de l'estimation des paramètres d'un modèle compartimental qui décrit la distribution postprandiale de l'azote alimentaire chez l'homme après l'ingestion d'un repas protéique. Un tel problème (i.e., un problème inverse classique) nécessite l'optimisation d'une fonctionnelle de coût mesurant la qualité de l'ajustement des prédictions du modèle à un jeu de données expérimentales. Dans notre cas particulier, les techniques classiques d'optimisation locale par méthodes de gradient s'avèrent incapables de trouver une solution satisfaisante au problème inverse, en raison du grand nombre de paramètres à estimer, de la forte non-linéarité de la fonctionnelle de coût et du peu de données expérimentales accessibles chez l'homme. Dans ce contexte, notre stratégie a consisté à développer une méthode de calibration qui utilise le maximum d'information disponible sur le comportement du système, de manière à diviser le problème inverse de grande taille en plusieurs sous-problèmes de taille plus réduite, pour lesquels une variante de la méthode du simplexe de NelderMead (NM) s'avère efficace. Cette méthode de calibration permet d'obtenir des valeurs proches des valeurs optimales pour la plupart des paramètres du modèle, que les données expérimentales injectées dans la fonction objectif soient bruitées ou non. A partir des valeurs de paramètres ainsi estimées, il est désormais possible de simuler de manière satisfaisante l'évolution temporelle de tous les compartiments du modèle physiologique, lequel constitue un outil permettant de décrire et d'expliquer les différents phénomenes dynamiques impliqués dans l'utilisation métabolique des protéines alimentaires chez l'homme.

${ }^{1}$ UMR 914 Nutrition Physiology and Ingestive Behavior, INRA-INAPG, 16 rue C. Bernard, Paris, 75005, France

${ }^{2}$ UMR 7598 Laboratoire Jacques-Louis Lions, CNRS, 175 rue du Chevaleret, Paris, 75013, France

(c) EDP Sciences, SMAI 2005 


\section{INTRODUCTION}

After ingestion of a meal, the assimilation of dietary protein is a complex process associated with a cascade of transient and dynamic metabolic events involved in controlling the distribution and metabolism of dietary amino acids (AA) and nitrogen (N) throughout the different $\mathrm{N}$ pools of the body (intestine, liver, muscles, etc.). During the postprandial phase (i.e., during the first 8 hours after a meal), dietary proteins are subjected to the following sequential metabolic processes. Ingested proteins entering the gastro-intestinal tract initially undergo digestion, releasing dietary AA and small peptides into the intestinal lumen. These AA and di- and tri-peptides are consecutively absorbed and transferred to the splanchnic organs, first the intestinal tissues and then the liver. Dietary $\mathrm{N}$ is then submitted to splanchnic uptake and metabolism for both anabolic and catabolic purposes: dietary AA are incorporated into the different intestinal and hepatic protein pools while they are concurrently deaminated in the liver and subsequently transferred to urea and ammonia, firstly in body water and secondly in urine. Dietary AA escaping to this splanchnic uptake are transferred via the blood circulation to peripheral organs such as muscles, where they also undergo metabolic uptake, either for oxidation or for anabolic purposes (muscular protein synthesis). An understanding of the kinetics and magnitude of these successive metabolic events is crucial to our knowledge of human nutrition. For example, it will allow the evaluation of dietary protein quality as a function of regional metabolic use, as well as the development of clinical management protocols for various health disorders through the application of specific nutritional strategies. However, little is still known, particularly in humans, about dietary $\mathrm{N}$ distribution, metabolism and regional utilization after protein ingestion, because of the dynamic nature of these physiological phenomena and the difficult or impossible experimental access to the main $\mathrm{N}$ pools involved in these processes. The many studies carried out in this field of research have mainly been based on isotopic methods enabling tracing of the metabolic fate of proteins or single AA in the few body pools accessible to experimental measurement $[5,10,24,33,37]$. However, these studies are generally conducted at steady state for practical reasons (i.e., during the fasting period when no proteins are administered or in an artificial fed state, where steady-state is achieved through the constant intragastric administration of a meal). Only a few studies have been conducted under physiological conditions in the fed state (i.e., the meal being ingested as a bolus) and have thus provided a valuable insight into the dynamic and adaptative features of protein metabolism [6,20,21].

In parallel with the increasing use of stable isotopes in human nutrition, the development of dynamic models to predict AA fluxes throughout different body $\mathrm{N}$ pools has markedly improved over the past 20 years. Particular attention has been paid to AA kinetics in the body, and numerous models have been developed to investigate the kinetics of leucine (an essential AA), as reported by Cobelli et al. [11] and Wolfe [40]. However, although some complex models have been proposed [7], they have only regarded the metabolism of one or a few AA. Only a limited number of studies have actually addressed the problem of dietary $\mathrm{N}$ modeling in humans, because of the broad, multiple exchange kinetics of $\mathrm{N}$ that make its study both practically and theoretically complex. In this context, a multi-compartmental model was recently developed by Fouillet et al. $[17,18]$ to describe the postprandial kinetics of the distribution and metabolism of dietary $\mathrm{N}$ in humans after the bolus ingestion of various protein meals. Compartmental modeling seems particularly well-suited to investigating such a complex system, because it entails reducing a markedly complex physiological system into a finite number of compartments and pathways, thus restricting the number of model variables and parameters. This simplification reduces mismatches between the complexity of the system and the limited data available from in vivo studies, especially in humans $[12,13]$.

Compartmental modeling has been widely used and validated in many areas of biological sciences $[1,2,8,27]$. In addition, there is a relatively complete analytic theory of linear compartmental systems [27], i.e., compartmental models whose parameters are constant or functions only of time. The theory and applications of compartmental modeling can be considered in terms of two main problems. The first is the development of plausible models for the processes at work in a particular biological system. This requires considerable background research in the field from which the problem arises: the structure and transfer pathways of the model should have meaning in terms of the known processes and structure of the real system. The second problem is a classic inverse problem: 
given such plausible models of a biological system, what experimental data should be collected and how should these data be used to determine the best model structure and estimate values for its parameters? The latter problem is known to be difficult to solve, and curbs the development of dynamic models which would promote functional understanding of the systems under consideration [31,34,42]. Calibration of the compartmental model proposed by Fouillet et al. [18] is not an exception to the rule. The aim of the present study is therefore to develop a robust calibration method able to estimate its parameter values with satisfactory precision.

This article is organized as follows: in the first part, we recall the main principles of compartmental modeling and present the mathematical description of the physiological problem. In the second part, we detail the search procedure that we have developed to estimate model parameters. In the last section, we present the numerical results that allowed us to validate our calibration method.

\section{Mathematical Description of the physiological PRoblem}

\subsection{Compartmental modeling}

\subsubsection{Definitions}

A compartmental model is made up of a finite number of macroscopic subunits called compartments that exchange material through different pathways.

A compartment is defined as a quantity of material acting kinetically as a homogeneous and distinct entity $[15,22,23]$. It should be distinguished from a physical volume or space, although the amount of material in such a volume may constitute a compartment. This amount of material can be expressed in terms of concentrations or total amounts, or sizes, of the compartments. Some compartments can be observed: the amount of material present in these compartments is measured at different points during an experiment. In our physiological context, a compartment represents the total amount of dietary $\mathrm{N}$ present either in a particular area of the human body (e.g. the dietary $\mathrm{N}$ present in the proximal intestinal lumen), or in a particular biochemical form (e.g. the dietary $\mathrm{N}$ present in body urea), or both (e.g. the dietary $\mathrm{N}$ present in a free AA form in the splanchnic area).

A transfer of material may represent a transformation (a chemical reaction, for instance) or a transport (a physical displacement of material from a compartment to another) and is characterized by a transfer coefficient, which can be constant or depend on time or the sizes of some compartments in the system [15]. In our physiological context, a transfer of dietary $\mathrm{N}$ represents either a transport (e.g. the transfer of dietary $\mathrm{N}$ through the compartments of the intestinal tract), or a biochemical transformation (e.g. the production of urea from the catabolism of AA in the liver), or a combination of both (e.g. the synthesis of protein from splanchnic free AA and its exportation into the blood circulation).

\subsubsection{Formulation and general theory}

Consider the $j^{t h}$ and $k^{t h}$ compartments of a model of $n$ compartments (Figure 1). Let $y_{j}(t)$ be the size of compartment $j$ at time $t$ and $y(t)$ the vector $\left(y_{j}(t)\right)_{1 \leq j \leq n}$. Let $F_{j k}(y, t)$ be the flow of material entering compartment $j$ from compartment $k$ at time $t, F_{0 j}(y, t)$ the direct flow of material out of the system from compartment $j$ at time $t$ and $F_{j 0}(t)$ the flow of material entering compartment $j$ from outside the system at time $t$. It should be noted that, except in very special cases, $F_{j 0}$ does not depend on the size of any model compartments. A compartment is governed by the instantaneous mass conservation law [27]:

$$
\forall j=1,2, \ldots, n, \forall t \geq 0, \frac{\mathrm{d} y_{j}}{\mathrm{~d} t}(t)=\sum_{k=1}^{n}\left(F_{j k}(y, t)-F_{k j}(y, t)\right)+F_{j 0}(t)-F_{0 j}(y, t)
$$




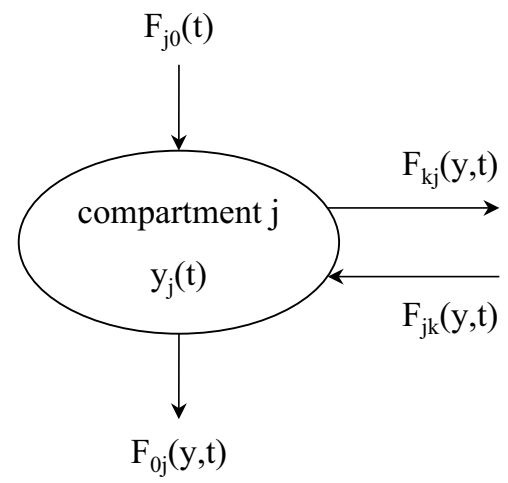

Figure 1. Example of diagram of the $j^{\text {th }}$ and $k^{\text {th }}$ compartments of an $n$-compartment model (using the notations described in section 1.1.2).

In most nutritional systems, the flow of material from a compartment $k$ to a compartment $j$ at time $t$ is supposed to be proportional to the amount of material present in the source compartment at that time [27]:

$$
\forall j=1,2, \ldots, n, \forall k=1,2, \ldots, n, \forall t \geq 0, \exists a_{j k}(y, t), F_{j k}(y, t)=a_{j k}(y, t) y_{k}(t) .
$$

Consequently, equation (1) can be written in the form:

$$
\forall j=1,2, \ldots, n, \forall t \geq 0, \frac{\mathrm{d} y_{j}}{\mathrm{~d} t}(t)=-\left(a_{0 j}(y, t)+\sum_{k=1}^{n} a_{j k}(y, t)\right) y_{j}(t)+\sum_{k=1}^{n} a_{j k}(y, t) y_{k}(t)+F_{j 0}(t) .
$$

$\left(a_{j k}\right)_{1 \leq j, k \leq n}$ are the transfer coefficients of the system and can be constant or depend on $y$ and/or on time. If all transfer coefficients are constant or functions only of time, such systems are called linear compartmental systems. If $f_{j j}(y, t)=-\left(f_{0 j}(y, t)+\sum_{k=1}^{n} f_{k j}(y, t)\right)$, we can now write (2) as equation (3):

$$
\forall j=1,2, \ldots, n, \forall t \geq 0, \frac{\mathrm{d} y_{j}}{\mathrm{~d} t}(t)=\sum_{k=1}^{n} a_{j k}(y, t) y_{k}(t)+F_{j 0}(t) .
$$

Let $A(y, t)$ be the matrix $\left(a_{j k}(y, t)\right)_{1 \leq j, k \leq n}$ and $F_{0}$ the vector $\left(F_{j 0}\right)_{1 \leq j \leq n}$, then:

$$
\forall t \geq 0, \frac{\mathrm{d} y}{\mathrm{~d} t}(t)=A(y, t) y(t)+F_{0}(t) .
$$

The compartmental system is thus described by a set of ordinary differential equations (ODEs), and knowing $A, y(0)$ and $F_{0}$, it is possible to simulate the temporal evolution of each state variable of the system. This is frequently called the "forward problem", i.e., the structure of the system is assumed to be known (number of compartments and transfer pathways between them), as are the values of its parameters, and the aim is to describe the kinetics of appearance and disappearance of material in each compartment of the model.

Of course, this is not the problem facing the biologist. The experimental scientist needs to design experiments and obtain data on the system under investigation, which are observations of the ODEs solution and from which one can deduce some structural properties of the model and estimate the values of its parameters. This inverse 
problem is difficult to solve and raises the following questions:

(i) which model structure is well adapted to both experimental data and our knowledge of the nutritional system;

(ii) given the set of experimental data, how can a model be calibrated to reproduce the experimental results in the best possible way?

\subsubsection{Model structure and mathematical description}

A 13-compartment model (Figure 2) has recently been developed to describe and simulate the postprandial distribution of dietary $\mathrm{N}$ in humans after ingestion of a protein meal. The choice of its structure (i.e., the answer to question (i) above) is detailed in [18].

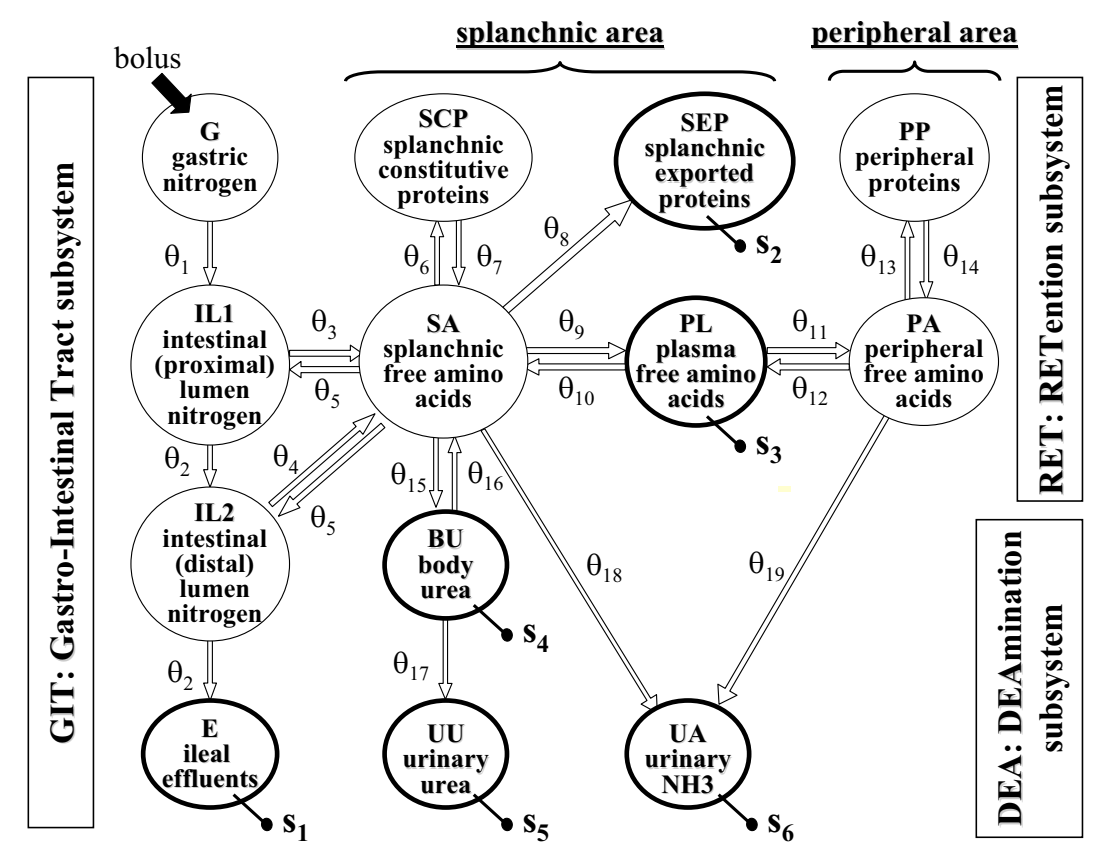

Figure 2. Compartmental model developped by Fouillet et al. [18], describing the postprandial distribution of dietary $\mathrm{N}$ in humans after ingestion of a protein meal. Circles indicate compartments representing kinetically distinct pools of dietary $\mathrm{N}$, arrows between them represent transfer pathways, associated with transfer rate constants in $\min ^{-1}\left(\theta_{i}\right.$ for $\left.i=1, \ldots, 19\right)$. Bullets indicate the sampled compartments. Samples $\mathrm{s}_{1}$ to $\mathrm{s}_{6}$ represent dietary $\mathrm{N}$ cumulative ileal effluents (E), splanchnic exported proteins (SEP), plasma free AA (PL), body urea (BU), cumulative urinary urea (UU) and cumulative urinary ammonia (UA), respectively. Data are expressed in permil of ingested $\mathrm{N}$.

This model consists of 13 compartments and 21 transfer pathways, associated with 19 transfer rate constants $\left(\theta_{i}\right.$ for $\left.i=1, \ldots, 19\right)$. The gastro-intestinal tract subsystem comprises four compartments representing dietary $\mathrm{N}$ in the stomach $(\mathrm{G})$, in the lumen of the proximal (IL1) and distal (IL2) small intestine, and at entry into the colon (E), respectively. Compartment $\mathrm{E}$ is experimentally monitored (sampling $\mathrm{s}_{1}$ ) every 30 minutes for eight hours after meal ingestion (detailed in [18]). The retention subsystem comprises six compartments representing dietary $\mathrm{N}$ in splanchnic free $\mathrm{AA}(\mathrm{SA})$, dietary $\mathrm{N}$ in splanchnic constitutive proteins ( $\mathrm{SCP}$ ), dietary $\mathrm{N}$ in splanchnic exported proteins (SEP), dietary $\mathrm{N}$ in plasma free AA (PL), dietary $\mathrm{N}$ in peripheral free AA (PA) and dietary $\mathrm{N}$ in peripheral proteins $(\mathrm{PP})$. Compartments SEP and PL are experimentally monitored (sampling 
$\mathrm{s}_{2}$ and $\mathrm{s}_{3}$, respectively) every 30 minutes for eight hours after meal ingestion (detailed in [18]). The deamination subsystem consists of three compartments representing dietary $\mathrm{N}$ in body urea (BU), dietary $\mathrm{N}$ in urinary urea (UU) and dietary $\mathrm{N}$ in urinary ammonia (UA). These three compartments are also experimentally monitored (sampling $\mathrm{s}_{4}, \mathrm{~s}_{5}$ and $\mathrm{s}_{6}$, respectively) every hour for eight hours after meal ingestion (detailed in [18]).

The model parameters $\theta_{i}$ are supposed to be constant for all $i=1, \ldots, 19$, i.e., they do not depend neither on time nor on the size of any compartment of the model. This means that the flow of material from a compartment to another is directly proportional to the amount of material available in the source compartment. It should be noted that the transfer rates from compartment IL1 to compartment IL2 and from compartment IL2 to compartment $\mathrm{E}$ are supposed to be equal for both simplicity and physiological reasons. The same is true for the transfer rates from compartment SA to compartments IL1 and IL2. Moreover, dietary N is assumed to enter the system as a bolus: $100 \%$ of dietary $\mathrm{N}$ is located in the stomach at time $t=0$, all the other compartments being empty at that time.

Let $\theta$ be the vector of model parameters: $\theta=\left(\theta_{1}, \ldots, \theta_{19}\right)$. Let $y(t, \theta)$ be the vector of state variables (compartment sizes) at time $t$ and $A(\theta)$ the corresponding compartmental matrix, represented below (Figure 3 ).

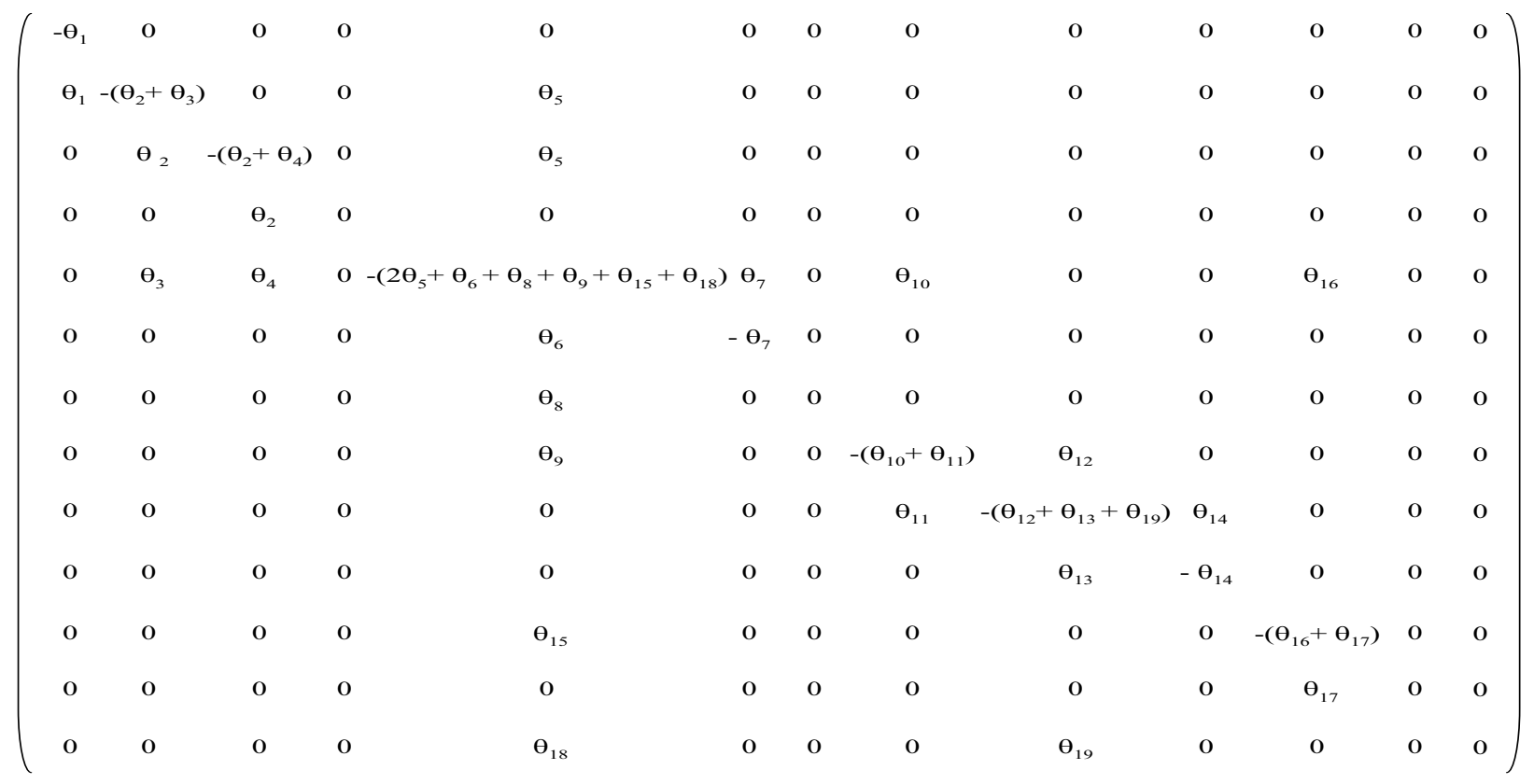

Figure 3. Compartmental matrix of the physiological model presented in Figure 2 and developed by Fouillet et al. [18].

The equation describing the temporal evolution of model compartments can be written as follows:

$$
\left\{\begin{array}{r}
\forall t \geq 0, \frac{\partial y(t, \theta)}{\partial t}=A(\theta) y(t, \theta) \\
y(0, \theta)=(1000,0, \ldots, 0)
\end{array}\right.
$$

since the amount of dietary $\mathrm{N}$ present in a compartment is expressed in permil of the ingested quantity. From here, since $y(0, \theta)$ does not depend on the vector of parameters $\theta$, it will be noted $y(0)$. The solution of equation (4) is therefore:

$$
\forall t \geq 0, y(t, \theta)=\exp (A(\theta) t) y(0) .
$$


Equation (5) provides an analytical formula describing the temporal evolution of all compartments of the model, which makes it possible to simulate the evolution of compartments that could not be observed experimentally, despite their metabolic and nutritional importance. In this study, we take the model structure of Figure 2 for granted and present a method to estimate its parameters (answer to the second question described above).

\subsection{Model calibration}

The calibration of a compartmental model consists in determining the parameter values that allow the fitting of model predictions to the experimental data obtained for all sampled compartments $[9,14,16,19,34]$. This constitutes an iterative process involving three steps:

(i) simulation of the model responses using a set of parameters from the search space;

(ii) evaluation of the quality of the model predictions (i.e., their ability to fit the experimental observations), quantified by an objective function;

(iii) improvement of the objective function value (by iteratively processing the two previous steps) until satisfactory performance is achieved or until further improvements become negligible.

\subsubsection{Objective functions used in compartmental modeling}

Let $n_{c}$ be the number of sampled compartments of the model and, for $j=1,2, \ldots, n_{c}$, let $n_{t}^{j}$ be the total number of sampling times for compartment $j$. The experimental error of measurement $e_{i j}$ made on the size of compartment $j$ at time $t_{i}$ is defined as follows:

$$
\forall j=1,2, \ldots, n_{c}, \forall i=1,2, \ldots, n_{t}^{j}, e_{i j}=z_{j}\left(t_{i}\right)-y_{j}\left(t_{i}, \theta\right),
$$

where $z_{j}\left(t_{i}\right)$ is the amount of material experimentally measured in the $j^{t h}$ compartment at time $t_{i}$ and $y_{j}\left(t_{i}, \theta\right)$ the size of compartment $j$ at time $t_{i}$ that is predicted by the model using the set of parameters $\theta$.

The objective function, which quantifies the quality of model predictions, is defined as a measure of the differences between predictions and observations [13,19]:

$$
\mathrm{F}=\sum_{j=1}^{n_{c}} \sum_{i=1}^{n_{t}^{j}} \omega\left(\epsilon_{i j}, \theta\right)
$$

where $\mathrm{F}$ is the objective function, $\omega$ an arbitrary loss function (usually the square or absolute value [19]) and $\epsilon_{i j}$ an appropriately weighted residual:

$$
\forall j=1,2, \ldots, n_{c}, \forall i=1,2, \ldots, n_{t}^{j}, \quad \epsilon_{i j}=\frac{z_{j}\left(t_{i}\right)-y_{j}\left(t_{i}, \theta\right)}{\sigma_{i j}},
$$

where $\sigma_{i j}$ is a positive weighting coefficient often related to the measurement error (its standard deviation).

By far the most widely used method is the least squares method which determines the values of parameters that minimize the sum of squares, generally weighted, of the differences between measured values and those calculated from the model. Its use is sanctioned by a long history of applications in many different fields and lengthy development of theory on linear parameter estimation (which means that the parameters appear linearly in the objective function). The theoretical justification for using least squares in linear parameter estimation was provided by the Gauss-Markov theorem [39]: assuming that the experimental errors are uncorrelated and have common variance, the least squares estimator is the one with minimum variance among all unbiased, linear estimators. This powerful background from linear estimation has strongly influenced work on nonlinear parameter estimation, but while there is a relatively complete theory for linear parameter estimation, that is 
not the case for nonlinear parameter estimation. Nonlinear least squares methods are often simply pragmatic methods sanctioned by long-standing use.

A more reliable theory for nonlinear parameter estimation is available: the maximum likelihood method [3,28]. This method consists in choosing the value of $\theta$ that maximizes the joint probability of having obtained the experimental data for all sampled compartments. For $j=1,2, \ldots, n_{c}$ and $i=1,2, \ldots, n_{t}^{j}$, let $p\left(\epsilon_{i j}, \theta\right)$ be the probability density function that the model prediction matches the observation of the $j^{\text {th }}$ compartment at time $t_{i}$. Thus, under the hypothesis of independent measurement errors, the likelihood function (LF) can be written as follows:

$$
\mathrm{LF}=\prod_{j=1}^{n_{c}} \prod_{i=1}^{n_{t}^{j}} p\left(\epsilon_{i j}, \theta\right)
$$

The experimental errors are supposed to be normally distributed. This assumption is fulfilled because the experimental errors are often composed of many small errors arising from the different steps of data acquisition, and according to the central limit theorem, the resulting error is normally distributed, even if contributing errors themselves may not be [32]. As a result, it has become common practice to assume that the normal distribution model is correct unless there is evidence to the contrary. The mathematical model that describes this assumption (i.e., that experimental measurements result in values that are normally distributed around the predicted value, with a standard deviation of $\sigma_{i j}$ ) is the following:

$$
\forall j=1,2, \ldots, n_{c}, \forall i=1,2, \ldots, n_{t}^{j}, \quad p\left(z_{j}\left(t_{i}\right), \theta, \sigma_{i j}\right)=\frac{1}{\sqrt{2 \pi} \sigma_{i j}} \exp \left(-\frac{1}{2}\left(\frac{z_{j}\left(t_{i}\right)-y_{j}\left(t_{i}, \theta\right)}{\sigma_{i j}}\right)^{2}\right),
$$

where $p\left(z_{j}\left(t_{i}\right), \theta, \sigma_{i j}\right)$ is the probability density function of having obtained the experimental value $z_{j}\left(t_{i}\right)$ for compartment $j$ at time $t_{i}$, which differs from the predicted value under $\left(z_{j}\left(t_{i}\right)-y_{j}\left(t_{i}, \theta\right)\right)$. Using these notations, the final form of the joint probability density function is given by:

$$
\begin{aligned}
\mathrm{LF} & =\prod_{j=1}^{n_{c}} \prod_{i=1}^{n_{t}^{j}} p\left(z_{j}\left(t_{i}\right), \theta, \sigma_{i j}\right) \\
& =\prod_{j=1}^{n_{c}} \prod_{i=1}^{n_{t}^{j}} \frac{1}{\sqrt{2 \pi} \sigma_{i j}} \exp \left(-\frac{1}{2}\left(\frac{z_{j}\left(t_{i}\right)-y_{j}\left(t_{i}, \theta\right)}{\sigma_{i j}}\right)^{2}\right) .
\end{aligned}
$$

\subsubsection{Choice of an objective function}

In order to facilitate implementation, we decided to work with the logarithm of the likelihood function (LLF), which leads to equation (7):

$$
\mathrm{LLF}=\ln (\mathrm{LF})=-\frac{n_{c} n_{t}^{j}}{2} \ln (2 \pi)-\sum_{j=1}^{n_{c}} \sum_{i=1}^{n_{t}^{j}} \ln \left(\sigma_{i j}\right)-\frac{1}{2} \sum_{j=1}^{n_{c}} \sum_{i=1}^{n_{t}^{j}}\left(\frac{z_{j}\left(t_{i}\right)-y_{j}\left(t_{i}, \theta\right)}{\sigma_{i j}}\right)^{2} .
$$

If the standard deviations $\sigma_{i j}$ were known, the first two terms in equation (7) were known constants and maximizing the logarithm of the likelihood function would be the same as minimizing the sum of squares of the appropriately weighted differences between experimental data and model predictions. In this case, the maximum likelihood method thus provides a theoretical basis for the least squares approach under the assumptions of independence and normal distribution of the experimental errors. 


\begin{tabular}{cc||cc}
\hline \hline Parameter & Value $\left(\mathrm{min}^{-1}\right)$ & Parameter & Value $\left(\mathrm{min}^{-1}\right)$ \\
\hline$\theta_{1}^{\star}$ & 0.01000170 & $\theta_{10}^{\star}$ & 0.00048372 \\
$\theta_{2}^{\star}$ & 0.00545349 & $\theta_{11}^{\star}$ & 0.17268261 \\
$\theta_{3}^{\star}$ & 0.01232039 & $\theta_{13}^{\star}$ & 0.00954823 \\
$\theta_{4}^{\star}$ & 0.01289907 & $\theta_{14}^{\star}$ & 0.01056087 \\
$\theta_{5}^{\star}$ & 0.00179097 & $\theta_{15}^{\star}$ & 0.00020175 \\
$\theta_{6}^{\star}$ & 0.05717149 & $\theta_{16}^{\star}$ & 0.04329578 \\
$\theta_{7}^{\star}$ & 0.00013830 & $\theta_{17}^{\star}$ & 0.00130912 \\
$\theta_{8}^{\star}$ & 0.01255922 & $\theta_{18}^{\star}$ & 0.00037141 \\
$\theta_{9}^{\star}$ & 0.03510541 & $\theta_{19}^{\star}$ & 0.00003931 \\
\hline
\end{tabular}

TABLE 1. Values chosen for the 19 parameters of the compartmental model developed by Fouillet et al. [18] and represented in Figure 2.

In this study, our aim is to develop a method to calibrate the compartmental model, whose structure is presented in Figure 2. We thus chose a set of parameters $\theta^{\star}$ (reported in Table 1), which we knew to be physiologically plausible [18], to simulate data in the six sampled compartments. Then, we developed a calibration method able to find again this initial parameter set $\theta^{\star}$, using the data previously simulated. Thanks to equation (5) and knowing $\theta^{\star}$, the model predictions are thus calculated every $\Delta t=10 \min$ until $T_{\text {final }}=480$ min for all model compartments, and especially for those that are experimentally monitored during the clinical experiment, i.e., the following values are calculated:

$$
\forall j=1,2, \ldots, n_{c}, \forall i=1,2, \ldots, \frac{T_{\text {final }}}{\Delta t}, \quad y_{j}\left(i \Delta t, \theta^{\star}\right)
$$

These model predictions, referred to as "noise-free" data $\left(d_{0}\right)$, are represented in Figures 4,5 and 6 for compartments E and SEP, PL and UA, and BU and UU, respectively. Then, according to equation (6), "experimental" data are simulated as follows:

$$
\forall j=1,2, \ldots, n_{c}, \forall i=1,2, \ldots, \frac{T_{\text {final }}}{\Delta t}, z_{j}(i \Delta t)=y_{j}\left(i \Delta t, \theta^{\star}\right)+e_{i j},
$$

where "experimental" errors $e_{i j}$ are normally distributed with a mean of zero and a standard deviation of $\sigma_{i j}$. In this study, we consider the standard deviation $\sigma_{i j}$ to be known and proportional to the size of compartment $j$ at time $i \Delta t$, the constant of proportionality being at first the same for all sampled compartments:

$$
\exists C, \forall j=1,2, \ldots, n_{c}, \forall i=1,2, \ldots, \frac{T_{\text {final }}}{\Delta t}, \quad \sigma_{i j}=C y_{j}\left(i \Delta t, \theta^{\star}\right) .
$$

In fact, uniform weights (i.e., $\sigma_{i j}$ set to the same value for all $j=1,2, \ldots, n_{c}$ and $i=1,2, \ldots, \frac{T_{\text {final }}}{\Delta t}$ ) do not seem to be appropriate, since the error variances probably differ for each sampled compartment. On the other hand, constant relative errors seem to be a more correct hypothesis, according the same magnitude in the objective function to all sampled compartments at all sampling times, which is of major interest to us since the sampled compartments of our compartment model are of very different sizes (Figures 4, 5 and 6). For practical reasons, $\sigma_{i j}$ is chosen to be proportional to the measured size of compartment $j$ at time $i \Delta t$. Equation (8) thus becomes:

$$
\forall j=1,2, \ldots, n_{c}, \forall i=1,2, \ldots, \frac{T_{f i n a l}}{\Delta t}, \quad \sigma_{i j}=C z_{j}(i \Delta t)
$$


By eliminating the constant terms in equation (7), we obtain:

$$
\widetilde{L L F}=-\sum_{j=1}^{n_{c}} \sum_{i=1}^{\frac{T_{\text {final }}}{\Delta t}}\left(\frac{z_{j}(i \Delta t)-y_{j}(i \Delta t, \theta)}{z_{j}(i \Delta t)}\right)^{2}
$$

Here two levels of noise are tested: $\sigma_{i j}=0.01 y_{j}\left(i \Delta t, \theta^{\star}\right)$ and $\sigma_{i j}=0.05 y_{j}\left(i \Delta t, \theta^{\star}\right)$, which provides two kinds of "experimental" data, referred to as "low-noise" data $\left(d_{1}\right.$ : experimental error of $\left.1 \%\right)$ and "high-noise" data $\left(d_{2}\right.$ : experimental error of $5 \%$ ), respectively (in practice, the measurement errors are considered to be generally less than $5 \%$ of the sampled compartment sizes). These data are represented in Figures 4,5 and 6 for compartments $\mathrm{E}$ and SEP, PL and UA, and BU and UU, respectively.
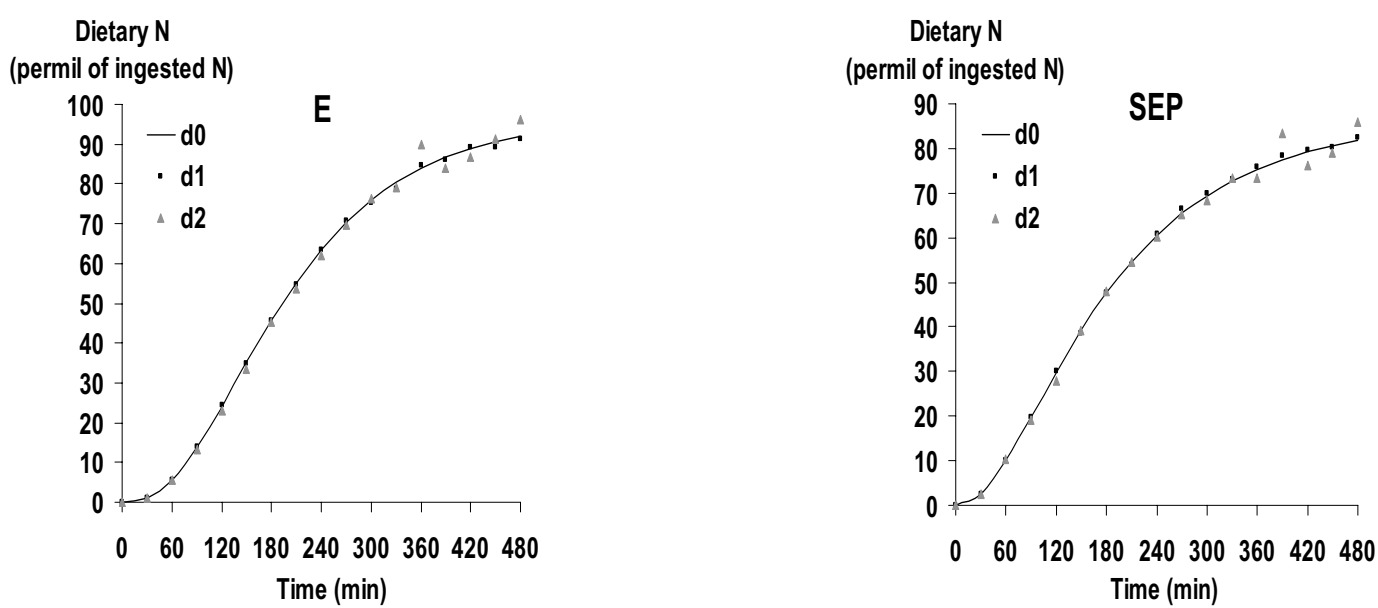

Figure 4. "Noise-free" $\left(d_{0}\right)$, "low-noise" $\left(d_{1}\right)$ and "high-noise" $\left(d_{2}\right)$ data simulated for compartments E and SEP of the physiological model represented in Figure 2, using the set of parameters $\theta^{\star}$ reported in Table 1 .

Finally, since maximizing expression (9) is equivalent to minimizing its opposite, our parameter estimation problem becomes:

$$
\min _{\theta \in\left(\mathbb{R}^{+}\right)^{n_{p}}} f(\theta) \quad \text { where } \quad f(\theta)=\sum_{j=1}^{n_{c}} \sum_{i=1}^{\frac{T_{\text {final }}}{\Delta t}}\left(\frac{z_{j}(i \Delta t)-y_{j}(i \Delta t, \theta)}{z_{j}(i \Delta t)}\right)^{2}
$$

The search space is $\left(\mathbb{R}^{+}\right)^{n_{p}}$ since the model parameters need by definition to be positive. Our parameter estimation problem thus amounts to minimizing the sum of squares of the differences between measured and predicted values, with a weight corresponding to the measured size of the compartment under consideration.

\subsubsection{Difficulties encountered}

The problem we address here is one of the global optimization of a highly nonlinear function in a high dimension. This kind of problem is known to be frequently ill-conditioned and multimodal. Traditional local, gradient-based optimization methods fail to find satisfactory solutions because they are frequently trapped in 

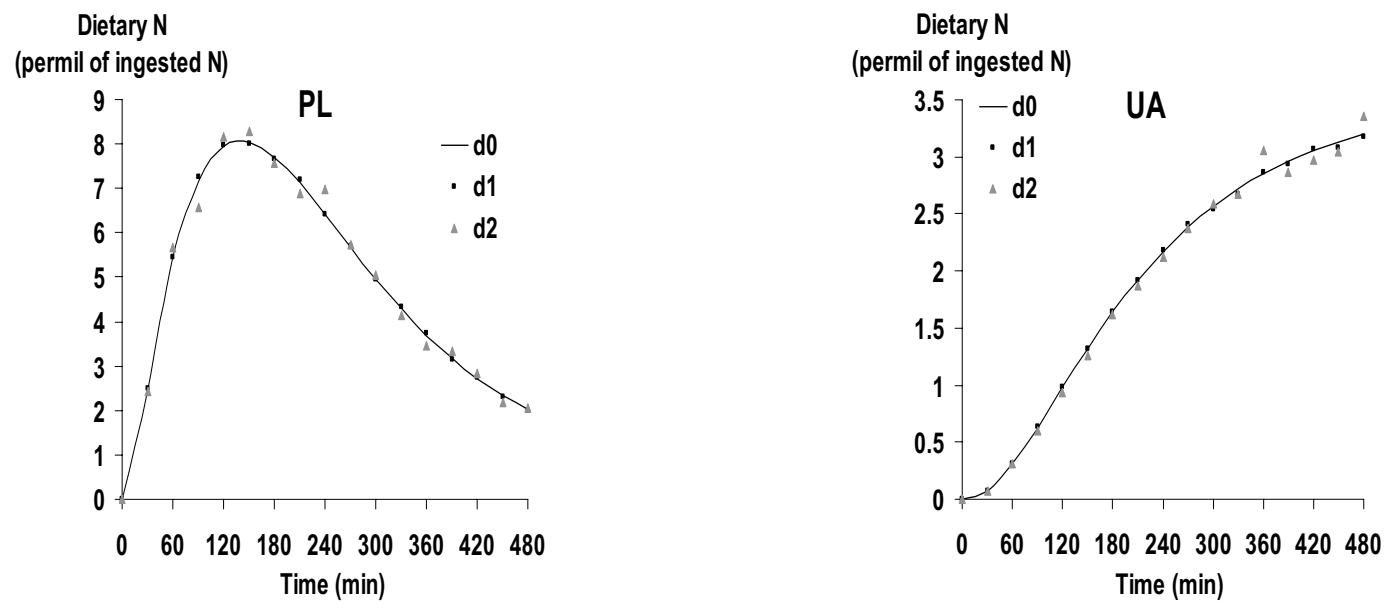

Figure 5. "Noise-free" $\left(d_{0}\right)$, "low-noise" $\left(d_{1}\right)$ and "high-noise" $\left(d_{2}\right)$ data simulated for compartments PL and UA of the physiological model represented in Figure 2, using the set of parameters $\theta^{\star}$ reported in Table 1 .
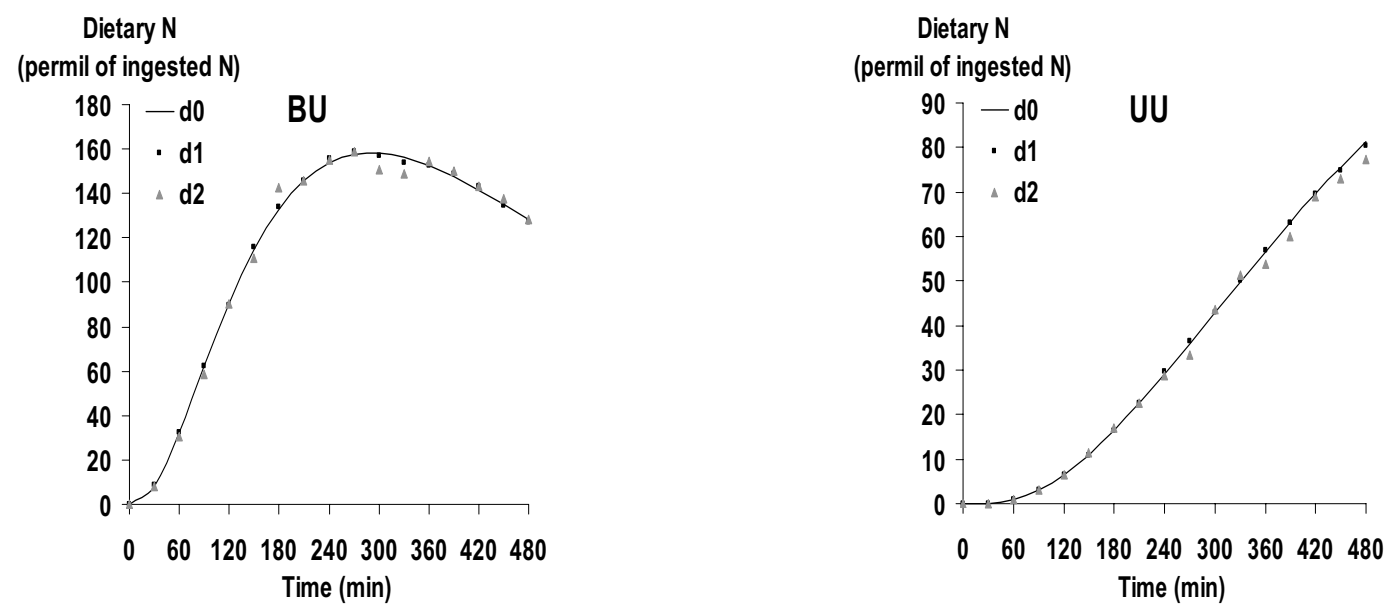

Figure 6. "Noise-free" $\left(d_{0}\right)$, "low-noise" $\left(d_{1}\right)$ and "high-noise" $\left(d_{2}\right)$ data simulated for compartments BU and UU of the physiological model represented in Figure 2, using the set of parameters $\theta^{\star}$ reported in Table 1 .

local optima instead of finding the global optimum. This problem even persists when the optimization method is associated with a multistart strategy, i.e., when the local method is used repeatedly by starting - randomly or not - from numerous initial sets of parameters, in order to visit the search space intensively. In the context of our parameter estimation problem, we first tested three optimization methods, which all failed to find the optimal set of parameters $\theta^{\star}$ :

(1) multistart + gradient-based method,

(2) multistart + simplex search method,

(3) genetic algorithm. 
We thus came to the conclusion that, in our particular case, the optimization problem is too complex to be properly solved by classic methods. In this study, the strategy used to overcome these difficulties consists in introducing as much information as possible about the physiological system in order to reduce the dimension of the search space to a minimum. This strategy is handled by carrying out a sensitivity analysis, which allows to quantitatively measure the sensitivity of system responses to changes in input parameters. We thus determine the model parameters that have the strongest influence on the observed compartments and use this information to break down the optimization problem into several simplier sub-problems of smaller dimensions, as described below.

\subsection{Sensitivity analysis}

\subsubsection{Theoretical sensitivity analysis}

A classic method to quantify the sensitivity of system responses to changes in input parameters is to calculate their sensitivity coefficients, that is the partial derivatives of the model state variables with respect to its parameters. This can be expressed as follows:

$$
\forall t \geq 0, \quad \nabla_{\theta} y(t, \theta)=\lim _{\Delta \theta \rightarrow 0} \frac{y(t, \theta+\Delta \theta)-y(t, \theta)}{\Delta \theta} .
$$

Knowing that the vector of state variables for the physiological model is given by equation (5), if $\Delta \theta$ is a small perturbation in the vector of parameters $\theta$, we have:

$$
\forall t \geq 0, y(t, \theta+\Delta \theta)=\exp (A(\theta+\Delta \theta) t) y(0)
$$

Since $A$ is linear in the vector of parameter $\theta$, then:

$$
\forall t \geq 0, y(t, \theta+\Delta \theta)=\exp ([A(\theta)+A(\Delta \theta)] t) y(0)
$$

Unfortunately, $A(\theta)$ and $A(\Delta \theta)$ do not commute and equation (10) can not be simplified. The sensitivity analysis is therefore conducted in a more practical way.

\subsubsection{Practical sensitivity analysis}

In this section, we define two criteria to evaluate the sensitivity coefficients of the physiological system. Let $\Delta \theta=\frac{\theta}{100}$ be a minor perturbation to the parameters and $\Delta t=10 \mathrm{~min}$ the time interval between two calculations of model responses.

a. Sensitivity analysis at the optimum set of parameters.

To measure the sensitivity of system responses to changes in parameter values from the optimum $\theta^{\star}$, we calculate the following quantities:

$$
\forall i=1,2, \ldots, \frac{T_{\text {final }}}{\Delta t}, \forall j=1,2, \ldots, n_{c}, \quad S_{i, j}^{\star}=\left|\frac{y_{j}\left(i \Delta t, \theta^{\star}+\Delta \theta^{\star}\right)-y_{j}\left(i \Delta t, \theta^{\star}\right)}{\Delta \theta^{\star}}\right| .
$$

These quantities allow us to quantify the influence on model responses of a small shift (1\%) of parameters from their optimum values. We can then evaluate the influence of each parameter, near the optimum, on the sampled compartments involved in calculating the objective function. 
b. Average sensitivity analysis.

To measure the average sensitivity of system responses to changes in input parameters, we randomly choose $N(N=1000)$ different sets of parameters $\left(\theta_{(k)}\right)_{1 \leq k \leq N}$ in the interval $\left[\frac{\theta^{\star}}{10}, 10 \theta^{\star}\right]$ and calculate the following quantities:

$$
\forall i=1,2, \ldots, \frac{T_{\text {final }}}{\Delta t}, \forall j=1,2, \ldots, n_{c}, \quad S_{i, j}=\frac{1}{N} \sum_{k=1}^{N}\left|\frac{y_{j}\left(i \Delta t, \theta_{(k)}+\Delta \theta_{(k)}\right)-y_{j}\left(i \Delta t, \theta_{(k)}\right)}{\Delta \theta_{(k)}}\right| .
$$

These quantities allow us to evaluate the influence on model responses of a minor perturbation of parameters from different sets of parameters, randomly chosen in the interval $\left[\frac{\theta^{\star}}{10}, 10 \theta^{\star}\right]$. We can thus quantify the average and also variability of the influence of each parameter on the sampled compartments involved in calculating the objective function.

\subsubsection{Results of the sensitivity analysis}

In this section, we detail the sensitivity analysis compartment by compartment, in order to specify the parameters with the strongest influence on each of the sampled compartments of the model. The results of the sensitivity analysis for compartment E and SEP are reported in Figure 7. They reveal that only four parameters $\left(\theta_{1}, \theta_{2}, \theta_{3}\right.$ and $\left.\theta_{4}\right)$ have a major influence on compartment $\mathrm{E}$, the other parameters being about 10 times less influential. Let $\theta^{(1)}$ be defined as follows: $\theta^{(1)}=\left(\theta_{1}, \theta_{2}, \theta_{3}, \theta_{4}\right)$. Besides the parameter set $\theta^{(1)}$, four parameters $\left(\theta_{6}, \theta_{8}, \theta_{9}\right.$ and $\left.\theta_{15}\right)$ have a major influence on compartment SEP, the other parameters being about 10 times less influential. Among these parameters, $\theta_{8}$ clearly stands out. Let $\theta^{(2)}$ be defined as follows: $\theta^{(2)}=\left(\theta_{8}\right)$.

E

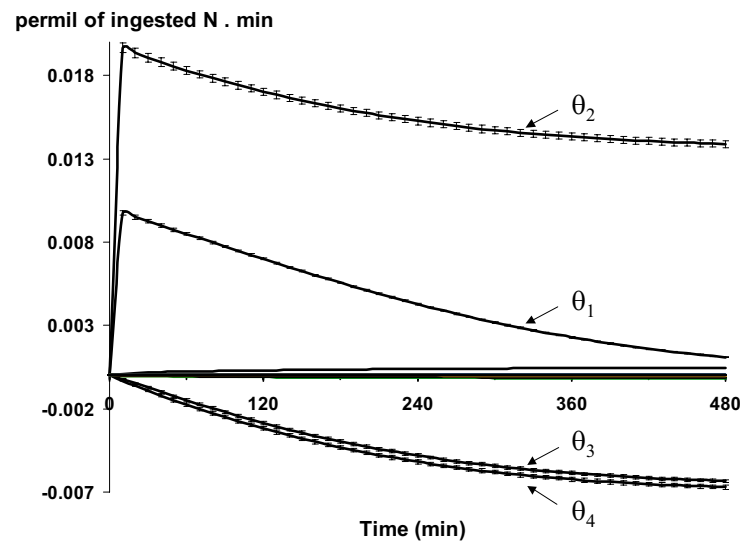

SEP

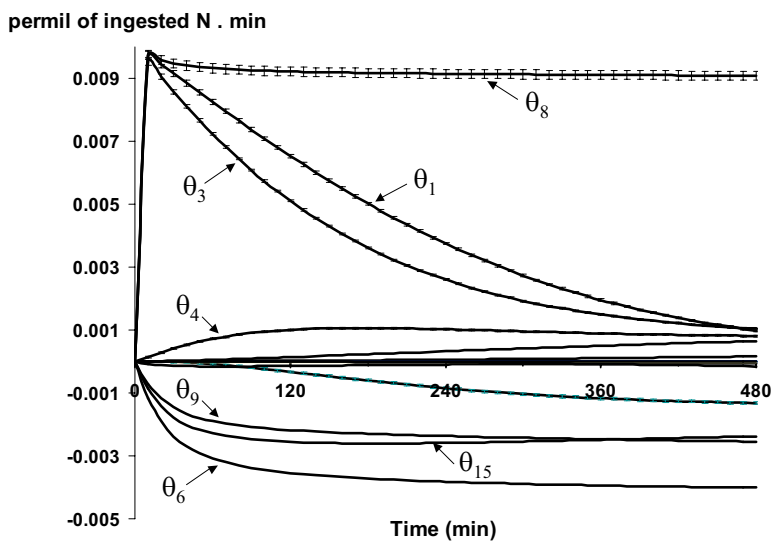

FIGURE 7. Sensitivity of model parameters to compartments E (ileal Effluents) and SEP (Splanchnic Exported Protein): sensitivity coefficients $S_{i, 1}^{\star}$ and $S_{i, 2}^{\star}$ calculated at the optimum $\theta^{\star}$ for $i=1,2, \ldots, \frac{T_{\text {final }}}{\Delta t}$, and their variability calculated from $N$ different sets of parameters in the interval $\left[\frac{\theta^{\star}}{10}, 10 \theta^{\star}\right]$, using the notations defined in section 1.3.2.

The results of the sensitivity analysis for compartment BU and UU are reported in Figure 8. They reveal that, besides the previous sets of parameters $\left(\theta^{(1)}, \theta^{(2)}\right)$, five parameters $\left(\theta_{6}, \theta_{9}, \theta_{15}, \theta_{16}\right.$ and $\left.\theta_{17}\right)$ have a major influence on compartment BU. Among these parameters, $\theta_{15}$ clearly stands out. $\theta^{(3)}$ is thus defined as follows: $\theta^{(3)}=\left(\theta_{15}\right)$. Then, besides the previous sets of parameters $\left(\theta^{(1)}, \theta^{(2)}, \theta^{(3)}\right)$, only two parameters $\left(\theta_{6}\right.$ and $\left.\theta_{17}\right)$ 

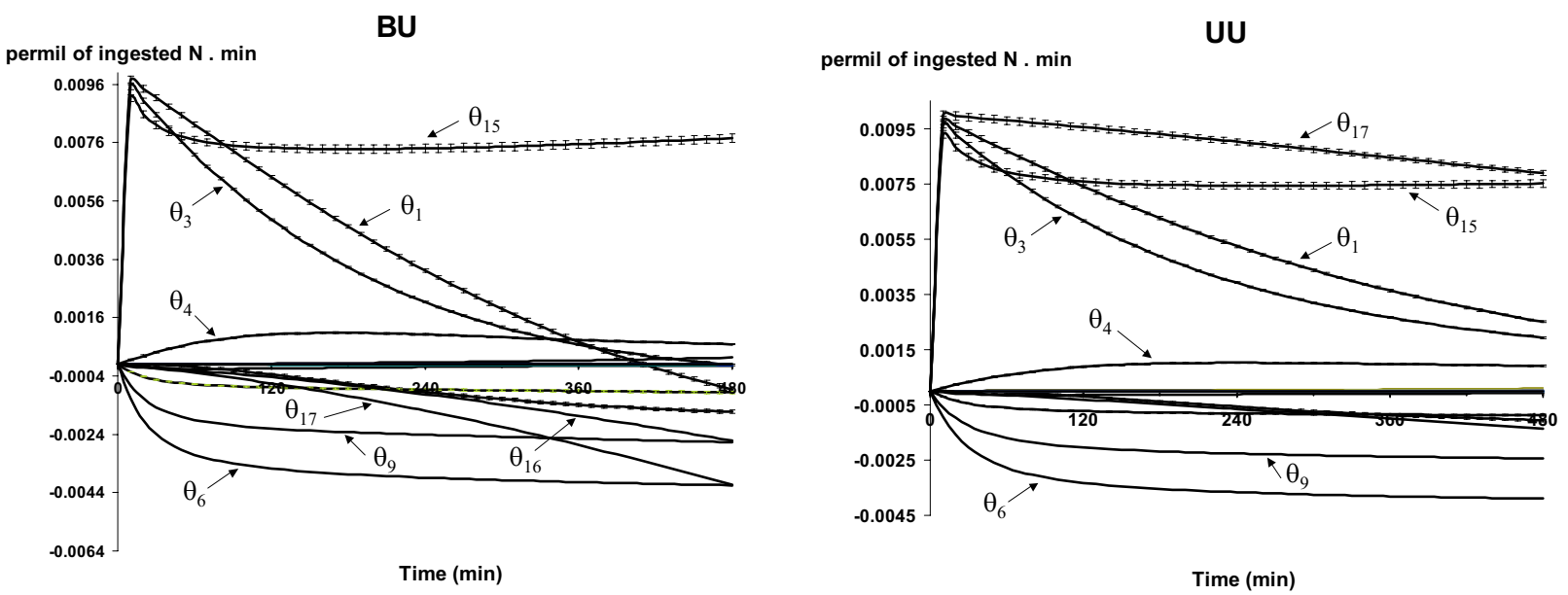

FIGURE 8. Sensitivity of model parameters to compartments BU (Body Urea) and UU (Urinary Urea): sensitivity coefficients $S_{i, 4}^{\star}$ and $S_{i, 5}^{\star}$ calculated at the optimum $\theta^{\star}$ for $i=1,2, \ldots, \frac{T_{f i n a l}}{\Delta t}$, and their variability calculated from $N$ different sets of parameters in the interval $\left[\frac{\theta^{\star}}{10}, 10 \theta^{\star}\right]$, using the notations defined in section 1.3.2.

have a major influence on compartment UU. Among these parameters, $\theta_{17}$ clearly stands out. Let $\theta^{(4)}$ be defined as follows: $\theta^{(4)}=\left(\theta_{17}\right)$.

The results of the sensitivity analysis for compartment PL and UA are reported in Figure 9.
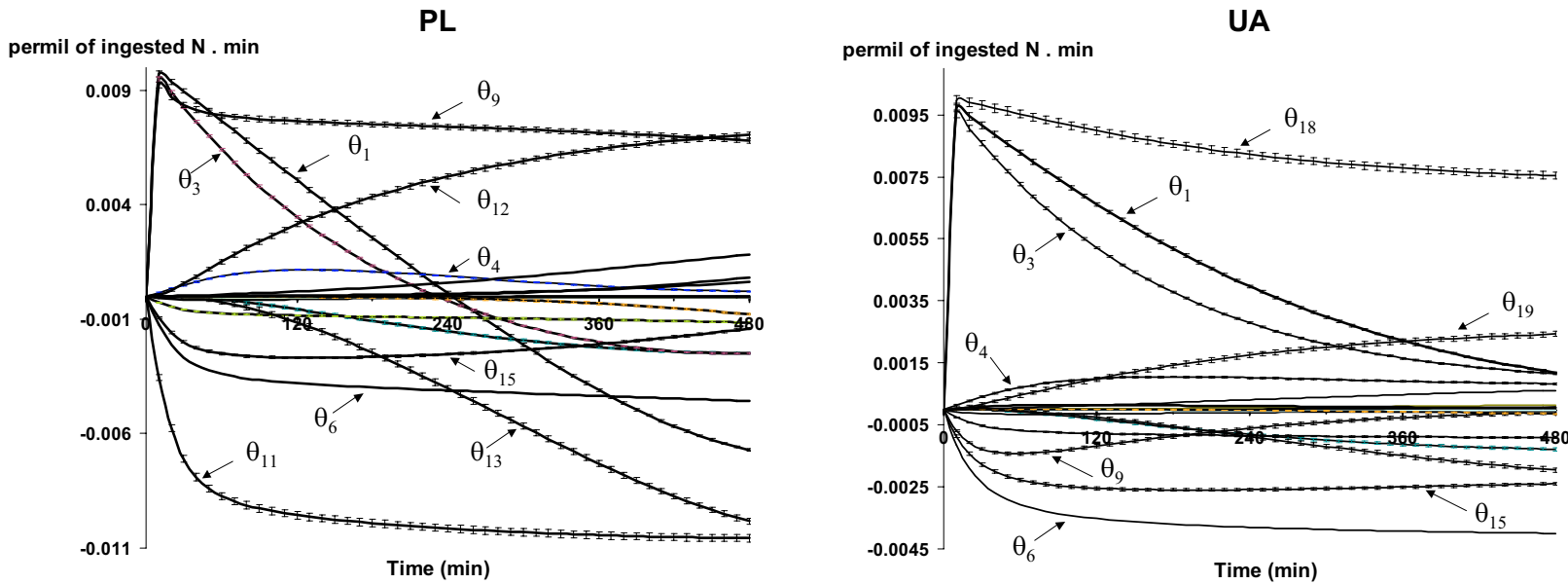

Figure 9. Sensitivity of model parameters to compartments PL (Plasma AA) and UA (Urinary Ammonia): sensitivity coefficients $S_{i, 3}^{\star}$ and $S_{i, 6}^{\star}$ calculated at the optimum $\theta^{\star}$ for $i=1,2, \ldots, \frac{T_{f i n a l}}{\Delta t}$, and their variability calculated from $N$ different sets of parameters in the interval $\left[\frac{\theta^{\star}}{10}, 10 \theta^{\star}\right]$, using the notations defined in section 1.3.2.

Besides the previous sets of parameters $\left(\theta^{(1)}, \theta^{(2)}, \theta^{(3)}, \theta^{(4)}\right)$, five parameters $\left(\theta_{6}, \theta_{9}, \theta_{11}, \theta_{12}\right.$ and $\left.\theta_{13}\right)$ have a major influence on compartment PL. $\theta^{(5)}$ is thus defined as follows: $\theta^{(5)}=\left(\theta_{9}, \theta_{11}, \theta_{12}, \theta_{13}\right)$. Finally, besides the 
previous sets of parameters $\left(\theta^{(1)}, \theta^{(2)}, \theta^{(3)}, \theta^{(4)}, \theta^{(5)}\right)$, three parameters $\left(\theta_{6}, \theta_{18}\right.$ and $\left.\theta_{19}\right)$ have a major influence on compartment UA. Let $\theta^{(6)}$ be defined as follows: $\theta^{(6)}=\left(\theta_{18}, \theta_{19}\right)$. Despite its influence on most of the sampled compartments, $\theta_{6}$ does not appear in the list of selected parameters (this will be explained below). Let $\theta^{(7)}$ denote the vector $\left(\theta_{5}, \theta_{6}, \theta_{7}, \theta_{10}, \theta_{14}, \theta_{16}\right)$ of the remaining parameters, which, except for $\theta_{6}$, exert a minor influence on the sampled compartments.

\section{Description of the Algorithm}

As previously explained, the calibration method that we developed consists in breaking down the whole optimization problem into several simplier sub-problems of smaller dimensions to be solved successively. By analogy with gradient-based optimization methods, our algorithm is designed to search the directions that ensure the most significant decrease of the objective functions associated with each sub-problem. The sensitivity analysis previously conducted allows us to focus only on the model parameters that lead to the greatest variations of the objective function values during the successive fitting processes, before estimating all parameters simultaneously during the final phase of the algorithm.

\subsection{Global description}

Using the previous notations, for $j=1,2, \ldots, n_{c}$ and $\theta$ a set of parameters, define $f_{j}(\theta)$ as:

$$
f_{j}(\theta)=\sum_{i=1}^{\frac{T_{f i n a l}}{\Delta t}}\left(\frac{z_{j}(i \Delta t)-y_{j}(i \Delta t, \theta)}{z_{j}(i \Delta t)}\right)^{2} .
$$

In our physiological problem, $n_{c}=6$ and $f_{1}, f_{2}, f_{3}, f_{4}, f_{5}$ and $f_{6}$ represent the sums over all sampling times of the relative errors between the "experimental" data and the model predictions for compartments E, SEP, BU, UU, PL and UA, respectively. The objective function thus becomes:

$$
f(\theta)=\sum_{j=1}^{n_{c}} f_{j}(\theta)=\sum_{j=1}^{n_{c}} \sum_{i=1}^{\frac{T_{f i n a l}}{\Delta t}}\left(\frac{z_{j}(i \Delta t)-y_{j}(i \Delta t, \theta)}{z_{j}(i \Delta t)}\right)^{2} .
$$

As shown in the previous section, each $f_{j}(\theta)$ can be written as follows:

$$
f_{j}(\theta)=f_{j}\left(\theta^{(1)}, \ldots, \theta^{(j)}, \theta^{(j+1)}, \ldots, \theta^{\left(n_{c}+1\right)}\right)
$$

where the sets of parameters $\left(\theta^{(1)}, \ldots, \theta^{(j)}\right)$ have a major influence on $f_{j}$, whereas the other parameters $\left(\theta^{(j+1)}\right.$, $\left.\ldots, \theta^{\left(n_{c}+1\right)}\right)$ are about 10 times less influential on this part of the objective function.

Since all the optimization methods previously tested failed to minimize $f$, we decided to introduce this information in our calibration method. The idea was to proceed as follows:

(1) Start by minimizing $f_{1}$, allowing only $\theta^{(1)}$ to vary (the other parameters being set at values randomly chosen within intervals of physiological values). $f_{1}$ is minimized using a multistart strategy: a local optimization algorithm (described below) is started from many initial values of $\theta^{(1)}$ randomly chosen in a large interval $I_{1}$, since we have no a priori idea of its optimum value. This provides many acceptable 
values for $\theta^{(1)}$, in particular a minimum and a maximum value for each model parameter included in $\theta^{(1)}$, which allows to markedly reduce the initial parameter space $I_{1}$ to a smaller search interval $\widetilde{I}_{1}$.

(2) Continue by minimizing $f_{1}+f_{2}$, using the same multistart strategy and allowing only $\left(\theta^{(1)}, \theta^{(2)}\right)$ to vary, in a search space $\left(\widetilde{I}_{1} \times I_{2}\right)$ that has been significantly restricted for the set of parameters $\theta^{(1)}$ in the course of step (1) of the algorithm (the other parameters being set at values randomly chosen within intervals of physiological values);

(3) Pursue by minimizing $\sum_{k=1}^{j} f_{k}$, with $2<j \leq n_{c}$, using the same multistart strategy and allowing only $\left(\theta^{(1)}, \ldots, \theta^{(j)}\right)$ to vary, in a search space $\left(\widetilde{I_{1}} \times \ldots \times \widetilde{I}_{j-1} \times I_{j}\right)$ that has been significantly restricted for the sets of parameters $\left(\theta^{(1)}, \ldots, \theta^{(j-1)}\right)$ in the course of the previous $(j-1)$ steps of the algorithm (the other parameters being set at values randomly chosen within intervals of physiological values);

(4) End by minimizing the complete objective function $f$, using the same multistart strategy and allowing all parameters to vary in a search space $\left(\widetilde{I}_{1} \times \ldots \times \widetilde{I}_{n_{c}}\right)$ that has been significantly restricted in the course of the previous $n_{c}$ steps of the algorithm for all influential model parameters, i.e., for the sets of parameters $\left(\theta^{(1)}, \ldots, \theta^{\left(n_{c}\right)}\right)$.

At intermediate step $j$ of this algorithm, the aim is to obtain values for $\left(\theta^{(1)}, \ldots, \theta^{(j)}\right)$ that are not too far from the optimum values, so that they can be used in subsequent steps of the algorithm as good starting points for the optimization procedure. During steps (2) and (3) of the algorithm, $\theta_{6}$ plays a particular role. Despite its non-negligeable influence on most of the sampled compartments, it can not be handled like any of the other influential parameters: in fact, when integrated in the optimization process and allowed to vary, this parameter makes the algorithm fail because of its strong correlation with other parameters such as $\theta_{8}, \theta_{9}, \theta_{15}$ and $\theta_{18}$, which are the main transfer rates around the central compartment SA. This affects the ability of our algorithm to find the optimum, and $\theta_{6}$ is therefore set at different values chosen in a narrow grid of the interval $\left[10^{-3}, 10^{-1}\right]$ (interval of physiologically plausible values) during steps $(2)$ and (3) of the algorithm. The last step of the algorithm is crucial and consists in minimizing $f$ on $\left(\mathbb{R}^{+}\right)^{n_{p}}$, from starting points that are already close to the optimum values for most of the model parameters, so that any local optimizer should be succesfull in its optimization process. The optimization method used at each step of the algorithm is a variant of the Nelder-Mead (NM) simplex search procedure and is described below.

\subsection{Development of a variant of the Nelder-Mead simplex search procedure}

The NM simplex search procedure is one of the most popular direct search method aimed at minimizing unconstrained real functions $[29,35]$. It is a derivative-free algorithm (i.e., that neither computes nor approximates the derivatives of the objective function), which is of particular interest when information on the gradient of the objective function is either unavailable or unreliable [30]. The simplex search method is based on geometrical principles: an initial simplex, that is a set of $n+1$ points of $\mathbb{R}^{n}$ (a triangle in $\mathbb{R}^{2}$, a tetrahedron in $\mathbb{R}^{3}$, etc.), is computed near an initial point specified by the user and new simplexes are iteratively generated through the geometrical operations of reflection, expansion, contraction and shrinkage [35] in order to find a point improving the objective function value.

In this study, the original NM simplex search algorithm [35] is used as a basis and made more complex by incorporating many of its desirable properties while avoiding some of its weaknesses - in particular, its sensitivity to starting values, its premature termination at a local minimum, and its lack of robustness against noisy responses [25]. Moreover, since the parameters of our metabolic system are bounded, the NM search procedure, originally conceived for unconstrained optimization, is here modified in order to take these constraints into account. From now on, this Variant of the NM search procedure is referred to as VNM algorithm. 


\subsubsection{Description of the VNM algorithm}

The main difference between the NM and VNM algorithms is that the new procedure operates in $P$ successive phases (instead of one for the NM simplex search). A previous study showed that restarting the NM procedure twice significantly improves the performances of the optimization algorithm [26]. In this study, we determine the largest integer $P$ that significantly improves some convergence properties of the simplex search procedure, when applied to our specific physiological purpose. Moreover, some parameters of the NM algorithm (size of the initial simplex, shrinkage coefficient) are modified at each phase of the VNM algorithm, which significantly improves its speed of converge, as described in [25].

The VNM algorithm operates in $P$ phases indexed by a phase counter $\varphi$. Let $n_{p}$ be the number of parameters to be estimated during optimization, then a simplex is a set of $n_{p}+1$ points of $\mathbb{R}^{n_{p}}$, or vertices. At the very beginning of the search procedure, the user provides an initial point that is the starting point for the overall search procedure as it becomes one vertex of the initial simplex and is used to construct the other vertices. At iteration $q$, within phase $\varphi$, the objective function is evaluated at each vertex $\theta_{i}^{(\varphi, q)}$ of the current simplex, yielding the values $f_{i}^{(\varphi, q)}=f\left(\theta_{i}^{(\varphi, q)}\right)$, for $i=1, \ldots, n_{p}$, which are sorted in increasing order. Let $\theta_{\text {min }}^{(\varphi, q)}$ denote the vertex of the current simplex yielding:

$$
f_{\min }^{(\varphi, q)}=f\left(\theta_{\min }^{(\varphi, q)}\right)=\min \left\{f_{i}^{(\varphi, q)}: 1 \leq i \leq n_{p}+1\right\}
$$

Each iteration $q$ of the search procedure consists in generating a new simplex from the current one via the geometrical operations of reflection, expansion, contraction, or shrinkage [26,35], in order to find new points improving the objective function value, until a termination criterion is satisfied:

$$
\left\{\begin{array}{l}
\max _{1 \leq i \leq n_{p}+1}\left\|\theta_{i}^{(\varphi, q)}-\theta_{\min }^{(\varphi, q)}\right\| \leq \eta_{1} \\
\max _{1 \leq i \leq n_{p}+1}\left\|f_{i}^{(\varphi, q)}-f_{\min }^{(\varphi, q)}\right\| \leq \eta_{2}
\end{array}\right.
$$

where $\eta_{1}$ et $\eta_{2}$ are user-specified tolerances. If condition (11) is satisfied, the termination point of the current phase is recorded. Let $\hat{\theta}(\varphi)$ be the final estimate of the optimal solution delivered at the end of phase $\varphi$. At the end of the algorithm, the final estimated optimum $\hat{\theta}(P)$, also referred to as $\hat{\theta}$, is the set of parameters which yields the best value for the objective function over the successive $P$ phases.

\subsubsection{Handling of constraints}

The NM search procedure is adapted so that it can take the parameter bounds into account. We first tested a variable change to force our parameters to stay positive. As it modified the configuration of the search space, we then prefered to penalize the objective function, as described below.

For all $j=1,2, \ldots, n_{p}$, let $\theta_{i n f, j}\left(\theta_{\text {sup }, j}\right)$ be the inferior (superior) bound of the $j^{\text {th }}$ parameter to be estimated and $\theta_{\text {mean }}$ the parameter set defined as follows:

$$
\forall j=1,2, \ldots, n_{p}, \quad \theta_{\text {mean }, j}=\frac{\theta_{i n f, j}+\theta_{\text {sup }, j}}{2} .
$$

The objective function is penalized to make it explode when any vertex of the simplex gets over the parameter bounds. This is implemented as follows:

$$
\forall j=1,2, \ldots, n_{p}, \quad\left\{\begin{array}{l}
\text { if } \theta_{i, j}^{(\varphi, q)}<\theta_{\text {inf }, j}, \text { then: } f_{i}^{(\varphi, q)} \leftarrow f_{i}^{(\varphi, q)}+10000, \\
\text { if } \theta_{i, j}^{(\varphi, q)}>\theta_{\text {sup }, j}, \text { then: } f_{i}^{(\varphi, q)} \leftarrow f_{i}^{(\varphi, q)}+10000,
\end{array}\right.
$$


considering the $i^{\text {th }}$ of the current simplex and using the notations previously defined. Thus, a parameter set that violates one of its bounds will never be selected in the course of the search procedure since it is associated to a poor value of the objective function.

However, if there is an attractive basin outside the feasible domain, this way of penalizing the objective function will not prevent the algorithm from being trapped near the frontier inside the domain. To compensate for this drawback, the algorithm is reorientated towards a feasible region every time that a parameter bound is violated. This is made possible by projection:

$$
\forall j=1,2, \ldots, n_{p}, \quad\left\{\begin{array}{l}
\text { if } \theta_{i, j}^{(\varphi, q)}<\theta_{i n f, j}, \text { then, set: } \theta_{i, j}^{(\varphi, q)}=\theta_{\text {mean }, j}, \\
\text { if } \theta_{i, j}^{(\varphi, q)}>\theta_{\text {sup }, j}, \text { then, set: } \theta_{i, j}^{(\varphi, q)}=\theta_{\text {mean }, j}
\end{array}\right.
$$

Finally, in order to make sure that the algorithm will not loop indefinitely, this operation is allowed to occur only a limited number of times.

\subsection{Validation of the VNM search procedure}

In this section, we describe the main criteria and optimization test problems used to evaluate and compare the VNM search procedure with the original NM algorithm, when applied to our specific physiological purpose. The results of both procedures are statistically analysed, as described below, in order to determine which modifications of the standard algorithm significantly improve its performances.

\subsubsection{Performance criteria}

Four criteria are defined to test and compare the performances of both NM and VNM procedures for each optimization test problem: (i) the number of function evaluations; (ii) the relative error on the objective function between its estimated and optimal values; (iii) the maximum relative error on parameters between their estimated and optimal values; (iv) the average relative error on parameters between their estimated and optimal values. No single criterion is sufficient to evaluate and compare the performances of both search procedures, but a combination of the four would provide a good idea of the respective merits of each algorithm.

a. Computational work.

To measure the computational work of both NM and VNM procedures, we calculate the number of function evaluations required by each procedure before it terminates and delivers the final estimates $f(\hat{\theta})$ and $\hat{\theta}$ :

$$
\mathrm{FE}=\text { number of function evaluations before termination. }
$$

The quantity FE is a measure of the computational time necessary for each search procedure before termination.

b. Ability to converge to the optimum value of the objective function.

To measure the accuracy of the final result delivered by each simplex search procedure on the estimation of the optimal value of the objective function, we define the following quantity:

$$
\mathrm{RE}^{f}=\left|\frac{f(\hat{\theta})-f^{\star}}{f^{\star}}\right|
$$

since we constructed the tested functions so that $f^{\star} \neq 0$. Each tested function is optimized a large number of times from different starting points (as detailed below). When averaged over all these replications, the quantity $\mathrm{RE}^{f}$ provides a measure of the ability of both algorithms to find a solution to the optimization problem under consideration, independently of the starting point. 
c. Ability to converge to the optimum parameter values.

Let $d$ be the dimension of the test problem under consideration, i.e., the search space dimension. To measure the accuracy of the final result delivered by each simplex search procedure on the estimate $\hat{\theta}_{j}$ of the optimum value $\theta_{j}^{\star}$, we define two criteria:

$$
\begin{aligned}
& -\mathrm{RE}_{\text {max }}^{\theta}=\max _{1 \leq j \leq d}\left|\frac{\hat{\theta}_{j}-\theta_{j}^{\star}}{\theta_{j}^{\star}}\right|, \\
& -\mathrm{RE}_{\text {mean }}^{\theta}=\frac{1}{d} \sum_{j=1}^{d}\left|\frac{\hat{\theta}_{j}-\theta_{j}^{\star}}{\theta_{j}^{\star}}\right|,
\end{aligned}
$$

since we constructed the tested functions so that $\theta_{j}^{\star} \neq 0$, for all $1 \leq j \leq d$. When averaged over all the replications of the optimization procedures, the quantities $\mathrm{RE} \underset{\max }{\stackrel{\theta}{a}}$ and $\mathrm{RE}_{\text {mean }}{ }^{\theta}$ give an idea of the ability of both algorithms to find the global optimum of the function under consideration, independently of the starting point.

\subsubsection{Description of the tested functions}

The optimization test problems used to examine the performances of the NM and VNM search procedures were chosen among standard optimization benchmarks and compartmental-type functions in order to:

(1) check on some standard optimization benchmarks (parabolas, exponentials, Rosenbrock, Shekel) that the number of function evaluations FE is not too greatly increased when a VNM procedure is used as the search algorithm;

(2) show that the VNM procedure significantly improves the performance criteria $\mathrm{RE}^{f}, \mathrm{RE}_{\text {max }}^{\theta}$ and $\mathrm{RE}_{\text {mean }}^{\theta}$ on compartmental-type functions.

In the next section, we describe some of these tested functions as well as the starting points used, the optimum value of the function under consideration and the point at which the optimum is achieved.

a. Example of standard optimization benchmarks tested: parabolas.

Here we present one of the standard optimization benchmarks that we tested: parabolas. We chose three levels of dimension for this function: $d=4, d=12$ and $d=24$, referred to as "low", "medium" and "high" dimensions, respectively. This choice is justified by the fact that, according to the literature, the simplex search procedure tends to perform well up to about dimension 10 [35]: the "low" and "medium" dimensions thus allow us to test the ability of the VNM procedure to minimize functions on search spaces of "low" (very inferior to 10) and "medium" (just above 10) dimensions. We also wanted to test its performances on a function of "high" dimension in order to move closer to the characteristics of our physiological problem (19 parameters to be estimated).

The parabolas are defined as follows:

$$
f(\theta)=\sum_{i=1}^{d}\left(f_{i}(\theta)\right)^{2}+1 \quad \text { where } \quad f_{i}(\theta)=1.5^{d / 2}\left(\theta_{i}-1\right)
$$

In each case, the optimum function value of $f$ is $f^{\star}=1$ and is achieved at $\theta^{\star}=(1, \ldots, 1)$. Figure 10 depicts the parabola of "low" dimension in two directions $\left(e_{1}, e_{4}\right)$. The parabolas have been optimized many times, from different starting points randomly chosen within the interval $[-5,5]^{d}$. 


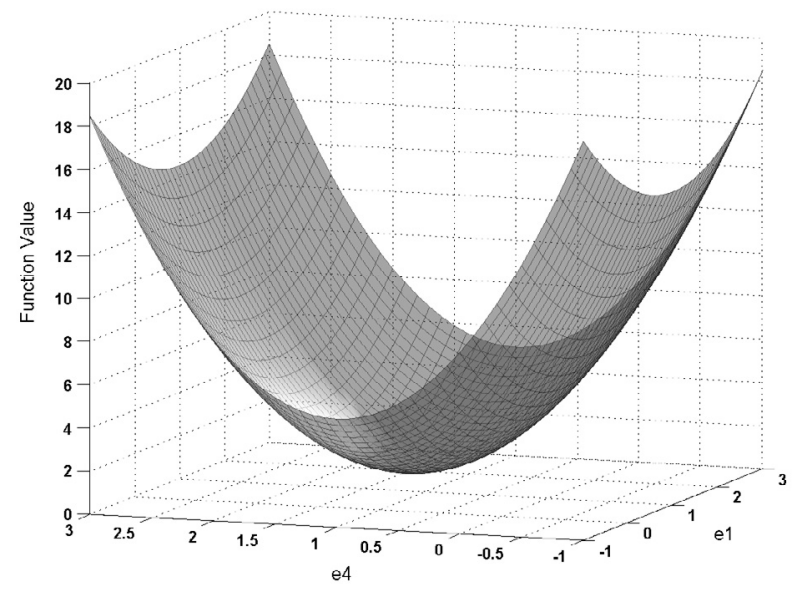

Figure 10. Plot of the parabola described in section 2.3.2 for $d=4$ in two directions $\left(e_{1}, e_{4}\right)$.

\section{b. Compartmental-type function.}

In this study, we tested both search procedures on the objective function of step (2) of the algorithm presented in section 2.1, in which we introduced different levels of gaussian noise by using the "experimental" data $d_{0}, d_{1}$ et $d_{2}$, defined in section 1.2.2. We chose to present the results obtained for this objective function because it is representative of the main difficulties we could encounter at the course of the calibration process (size of the search space, noise in data). Using the notations defined in section 2.1, the objective function to be minimized is as follows:

$$
\sum_{j=1}^{2} f_{j}\left(\theta^{(1)}, \theta^{(2)}, \theta^{(3)}, \ldots, \theta^{\left(n_{c}+1\right)}\right) .
$$

For $j>2$, we set $\theta^{(j)}$ to its optimum value $\theta^{\star(j)}$ and consider the following minimization problem:

$$
\begin{aligned}
\min _{\theta} f(\theta) \quad \text { with } \quad f(\theta) & =1+\sum_{j=1}^{2} f_{j}\left(\theta^{(1)}, \theta^{(2)}, \theta^{\star(3)}, \ldots, \theta^{\star\left(n_{c}+1\right)}\right), \\
\text { and } \quad f_{j}(\theta) & =\sum_{i=1}^{\frac{T_{\text {final }}}{\Delta t}}\left(\frac{z_{j}(i \Delta t)-y_{j}(i \Delta t, \theta)}{z_{j}(i \Delta t)}\right)^{2},
\end{aligned}
$$

where the "experimental" data $z_{j}$ are either $d_{0}, d_{1}$ or $d_{2}$. The optimal function value of $f$ is $f^{\star}=1$, $f^{\star}=1.008086186369$ or $f^{\star}=1.10796668076$, when the "experimental" data injected into the objective function are $d_{0}, d_{1}$ or $d_{2}$, respectively. In each case, the optimum function value is achieved at $\theta^{\star}=\left(\theta_{1}^{\star}, \theta_{2}^{\star}, \theta_{3}^{\star}, \theta_{4}^{\star}, \theta_{8}^{\star}\right)$, whose values are reported in Table 1 . Figure 11 depicts this function in two directions $\left(e_{1}, e_{8}\right)$ for data $d_{0}$. This objective function has been optimized a large number of times, from different starting points chosen on a grid of the interval $\left[10^{-3}, 5.10^{-2}\right]^{5}$. Here is tested the ability of both search algorithms to minimize the objective function of step (2) of the algorithm, knowing the exact values of the parameters not estimated at that step. 


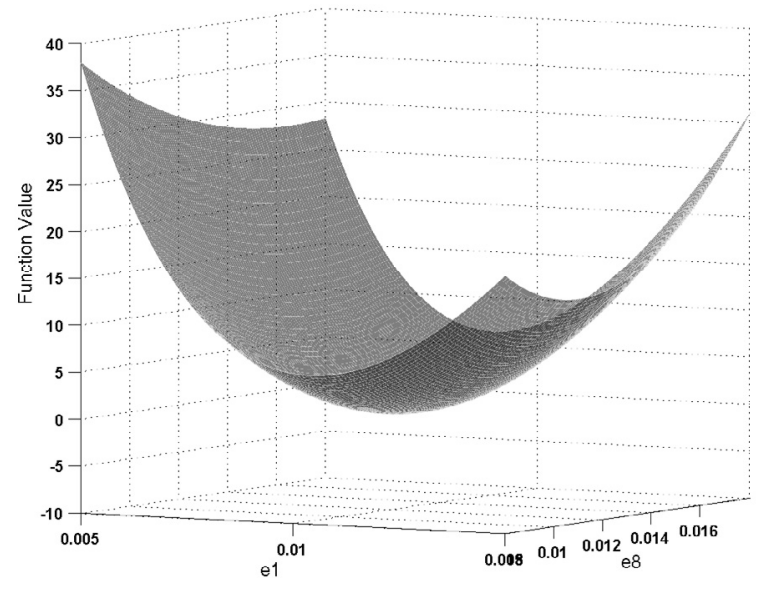

Figure 11. Plot of the compartmental-type function described in section 2.3.2 in the case of "noise-free" data $\left(d_{0}\right)$ in two directions $\left(e_{1}, e_{8}\right)$.

\subsubsection{Statistical analysis}

a. Statistical analysis on FE.

Results for FE values obtained with both procedures in the case of the standard optimization benchmarks are statistically tested in order to determine if FE is significantly affected by the search procedure used as the optimization algorithm. Each search procedure gives different results for FE depending on three factors of variation:

(i) $A_{i}$, the $i^{t h}$ level of optimization algorithm (NM for $i=1$ and VNM for $i=2, \ldots, 10$ where $i$ represents the number of phases $P$ of the search algorithm)

(ii) $D_{j}$, the $j^{t h}$ level of dimension ( 4 for $j=1,12$ for $j=2$ and 24 for $j=3$ )

(iii) $I P_{k}$, the $k^{\text {th }}$ replication of the treatment $\left(A_{i}, D_{j}\right)$, for $k=1, \ldots, 1000$ (multi-start).

A linear mixed model is postulated:

$$
\begin{gathered}
\forall i, \quad 1 \leq i \leq 10, \forall j, 1 \leq j \leq 3, \forall k, \quad 1 \leq k \leq 1000 \\
\mathrm{FE}_{i j k}=\alpha_{0}+\alpha_{A_{i}}+\alpha_{D_{j}}+\alpha_{A_{i} D_{j}}+\alpha_{I P_{k}}+\alpha_{D_{j} I P_{k}}+\epsilon_{i j k},
\end{gathered}
$$

where $\alpha_{A_{i}}, \alpha_{D_{j}}$ and $\alpha_{A_{i} D_{j}}$ are the contributions on FE of the fixed effects of the chosen algorithm, of the dimension of the objective function and of the interaction between both, respectively, whereas $\alpha_{I P_{k}}$ and $\alpha_{D_{j} I P_{k}}$ are the contributions of the random effects of the initial starting point and of the interaction between the level of dimension and the initial starting point, respectively. The statistical analysis of model (15) was performed using SAS software [36].

b. Statistical analysis on $R E^{f}, R E_{\text {max }}^{\theta}$ and $R E_{\text {mean }}^{\theta}$.

Similarly, results for $\mathrm{RE}^{f}, \mathrm{RE}_{\text {max }}^{\theta}$ and $\mathrm{RE}_{\text {mean }}^{\theta}$ values obtained for the compartmental-type functions are statistically tested in order to determine if these criteria are significantly affected by the search procedure used as the optimization algorithm. Each search procedure gives different results for $\mathrm{RE}^{f}, \mathrm{RE}_{\text {max }}^{\theta}$ and $\mathrm{RE}_{\text {mean }}^{\theta}$ depending on three factors of variation:

(i) $A_{i}$, the $i^{\text {th }}$ level of optimization algorithm (NM for $i=1$ and VNM for $i=2, \ldots, 10$ where $i$ represents the number of phases $P$ of the search algorithm)

(ii) $N_{j}$, the $j^{\text {th }}$ level of noise ("noise-free" for $j=1$, "low-noise" for $j=2$ and "high-noise" for $j=3$ ) 
(iii) $I P_{k}$, the $k^{\text {th }}$ replication of the treatment $\left(A_{i}, N_{j}\right)$, for $k=1, \ldots, 1000$ (multi-start).

A linear mixed model is postulated:

$$
\begin{gathered}
\forall i, \quad 1 \leq i \leq 10, \quad \forall j, \quad 1 \leq j \leq 3, \quad \forall k, \quad 1 \leq k \leq 1000, \\
\mathrm{Cr}_{i j k}=\alpha_{0}+\alpha_{A_{i}}+\alpha_{N_{j}}+\alpha_{A_{i} N_{j}}+\alpha_{I P_{k}}+\alpha_{N_{j} I P_{k}}+\epsilon_{i j k},
\end{gathered}
$$

where $\mathrm{Cr}$ is one of the criteria $\mathrm{RE}^{f}, \mathrm{RE}_{\text {max }}^{\theta}$ and $\mathrm{RE}_{\text {mean }}^{\theta}$, and $\alpha_{A_{i}}, \alpha_{N_{j}}$ and $\alpha_{A_{i} N_{j}}$ are the contributions of the fixed effects of the chosen algorithm, of the noise introduced in the data injected into the objective function and of the interaction between both, respectively, whereas $\alpha_{I P_{k}}$ and $\alpha_{N_{j} I P_{k}}$ are the contributions of the random effects of the initial starting point and of the interaction between the level of noise and the initial starting point, respectively. The statistical analysis of model (16) was performed using SAS software [36] on the logarithm of each criterion. This transformation is made necessary for numerical reasons, since the values of $\mathrm{RE}^{f}, \mathrm{RE}_{\max }^{\theta}$ and $\mathrm{RE}_{\text {mean }}^{\theta}$ are too small to be distinguished by the software.

\section{Results}

\subsection{Comparison between NM and VNM search procedures and choice of the optimization method}

The statistical results obtained regarding the different performance criteria are summarized in Table 2 and Table 3 and analysed in the following sections.

\subsubsection{Computational work (FE criterion)}

The results of the statistical tests performed on the performance criterion FE are presented in Table 2 for the parabolas, all levels of dimension taken together.

\begin{tabular}{lcc}
\hline \hline Procedure & FE & $\mathrm{Gr}^{\star}$ \\
\hline NM & 20779 & 1 \\
VNM $(P=2)$ & 44880 & 2 \\
VNM $(P=3)$ & $\mathbf{4 0 9 0 1}$ & $\mathbf{3}$ \\
VNM $(P=4)$ & 38669 & 3 \\
VNM $(P=5)$ & 39923 & 3 \\
VNM $(P=6)$ & 39380 & 3 \\
VNM $(P=7)$ & 39014 & 3 \\
VNM $(P=8)$ & 38732 & 3 \\
VNM $(P=9)$ & 40299 & 3 \\
VNM $(P=10)$ & 42502 & 3 \\
\hline
\end{tabular}

TABLE 2. Comparison between NM and VNM search procedures for performance criterion FE on the parabolas, all levels of dimension taken together. ${ }^{\star}$ Grouping of procedures with non-significant differences in performance (mixed models, $\mathrm{p}$ value $<0.05$ ).

In terms of the number of function evaluations performed by each search algorithm before delivering its final estimates, Table 2 shows that, in general, the NM procedure requires significantly less computational work than the VNM procedures. In fact, the number of function evaluations FE is doubled at the very worst when a VNM procedure is used as the optimization algorithm. On the other hand, there are generally no significant 
differences between the VNM procedures depending on their number of phases $P$. This means in particular that the computational work they require is not significantly increased with $P$, and thus an improvement in their performance is not to the detriment of their speed of convergence. Furthermore, the performance criterion FE can decrease with the number of phases $P$ : in the case of the parabolas, the number of function evaluations significantly decreases when the VNM procedure operates in 3 versus 2 phases. These results can be explained by the choice of the size of the initial simplex at the beginning of each phase of the search procedure. In a VNM procedure, the initial simplex computed at the very beginning of the first phase is quite large (50\%-increase in the initial value of each parameter) in order to make the algorithm visit a large domain of the search space, whereas the initial simplex computed at the very beginning of the last phase is quite small (5\%-increase in the initial value of each parameter) in order to refine the search and make the algorithm act like a local optimizer at this final stage. The size of the initial simplex then decreases linearly over successive phases so that the algorithm searches progressively less and less globally and more and more locally in the search space. In the case of the parabolas, increasing the number of phases of the VNM procedure, and thus computing simplices of intermediate sizes, seem to improve the speed of convergence of the algorithm. The results are similar in the case of the other standard optimization benchmarks (exponentials, Rosenbrock, Shekel) that we tested.

\subsubsection{Ability to converge to the optimum value of the objective function (RE $E^{f}$ criterion)}

The results of statistical tests performed on the performance criterion $\mathrm{RE}^{f}$ are presented in Table 3 for the compartmental-type function, whatever the noise level (i.e., for all levels of noise taken together). These results make it possible to both determine which procedure (NM or VNM) better converges to the optimum value of the objective function (i.e., provides the lower values of $\mathrm{RE}^{f}$ ), and which value for $P$ is the minimum number of phases of the VNM procedure providing the lower values of $\mathrm{RE}^{f}$ versus higher values of $P$ that do not significantly improve this performance criterion. In fact, VNM procedures provide values of $\mathrm{RE}^{f}$ that are always significantly smaller (and thus better) than those produced by the NM algorithm. In addition, VNM procedures yield values for $\mathrm{RE}^{f}$ that decrease with the number of phases $P$, this reduction being statistically significant up to $P=4$ : for $P>4$, there is no significant improvement in the ability of the VNM procedure to converge to the optimum value of the compartmental-type function.

3.1.3. Ability to converge to the optimum values of the parameters ( $R E_{\text {max }}^{\theta}$ and $R E_{\text {mean }}^{\theta}$ criteria)

Similarly, the results of statistical tests performed on performance criteria $\mathrm{RE}_{\text {max }}^{\theta}$ and $\mathrm{RE}_{\text {mean }}^{\theta}$ are presented in Table 3 for the compartmental-type function, for all levels of noise taken together.

\begin{tabular}{|c|c|c|c|c|c|c|c|}
\hline Procedure & $\log \left(\mathrm{RE}^{f}\right)$ & $\mathrm{Gr}^{\star}$ & Procedure & $\log \left(\mathrm{RE}_{\max }^{\theta}\right)$ & $\mathrm{Gr}^{\star}$ & $\log \left(\mathrm{RE}_{\text {mean }}^{\theta}\right)$ & $\mathrm{Gr}^{\star}$ \\
\hline $\mathrm{NM}$ & -5.283 & 1 & NM & -3.157 & 1 & -3.420 & 1 \\
\hline $\operatorname{VNM}(P=2)$ & -5.377 & 2 & VNM $(P=2)$ & -3.198 & 2 & -3.464 & 2 \\
\hline $\operatorname{VNM}(P=3)$ & -5.399 & 3 & $\operatorname{VNM}(P=3)$ & -3.202 & 2 & -3.467 & 2 \\
\hline VNM $(P=4)$ & -5.420 & 4 & $\operatorname{VNM}(P=4)$ & -3.218 & 2 & -3.483 & 2 \\
\hline $\operatorname{VNM}(P=5)$ & -5.431 & 4 & $\operatorname{VNM}(P=5)$ & -3.217 & 2 & -3.481 & 2 \\
\hline $\operatorname{VNM}(P=6)$ & -5.436 & 4 & $\operatorname{VNM}(P=6)$ & -3.229 & 2 & -3.494 & 2 \\
\hline $\operatorname{VNM}(P=7)$ & -5.445 & 4 & $\operatorname{VNM}(P=7)$ & -3.219 & 2 & -3.484 & 2 \\
\hline $\operatorname{VNM}(P=8)$ & -5.458 & 4 & $\operatorname{VNM}(P=8)$ & -3.225 & 2 & -3.489 & 2 \\
\hline $\operatorname{VNM}(P=9)$ & -5.452 & 4 & $\operatorname{VNM}(P=9)$ & -3.228 & 2 & -3.492 & 2 \\
\hline $\operatorname{VNM}(P=10)$ & -5.460 & 4 & $\operatorname{VNM}(P=10)$ & -3.236 & 2 & -3.500 & 2 \\
\hline
\end{tabular}

TABLE 3. Comparison between NM and VNM search procedures for performance criteria $\log \left(\mathrm{RE}^{f}\right), \log \left(\mathrm{RE}_{\text {max }}^{\theta}\right)$ and $\log \left(\mathrm{RE}_{\text {mean }}^{\theta}\right)$ on the compartmental-type function, all levels of noise taken together. ${ }^{\star}$ Grouping of procedures with non-significant differences in performance (mixed models, p value $<0.05$ ). 
These results make it possible to both determine which procedure (NM or VNM) better converges to the optimum (i.e., provides the lower values of $\mathrm{RE}_{\text {max }}^{\theta}$ and $\mathrm{RE}_{\text {mean }}^{\theta}$ ) and which value for $P$ is the minimum number of phases of the VNM procedure providing the lower values of $\mathrm{RE}_{\max }^{\theta}$ and $\mathrm{RE}_{\text {mean }}^{\theta}$ versus higher values of $P$ that do not significantly improve these performance criteria. VNM procedures provide values for $\mathrm{RE}_{\text {max }}^{\theta}$ and $\mathrm{RE}_{\text {mean }}^{\theta}$ that are significantly smaller (and thus better) than those produced by the NM algorithm. On the other hand, increasing the number of phases $P$ in the VNM procedure does not significantly improve these performance criteria. In fact, for $P>2$, there is no significant improvement in the ability of the VNM procedure to converge to the optimum set of parameters of the compartmental-type objective function.

In view of all these statistical results on the different performance criteria tested, we decided to choose a VNM search procedure operating in 4 phases as the optimization algorithm for our calibration method.

\subsection{Performance of the calibration method}

\subsubsection{Noise-free data $\left(d_{0}\right)$}

The results of the calibration method obtained when "noise-free" data are injected into the objective function, are presented in Table 4 .

\begin{tabular}{cccc}
\hline \hline Parameter & Optimum value $\left(\mathrm{min}^{-1}\right)$ & Estimated value $\left(\mathrm{min}^{-1}\right)$ & Relative Error $(\%)$ \\
\hline$\theta_{1}$ & 0.01000170 & 0.01000184 & 0.00138 \\
$\theta_{2}$ & 0.00545349 & 0.00545346 & 0.00047 \\
$\theta_{3}$ & 0.01232039 & 0.01232027 & 0.00097 \\
$\theta_{4}$ & 0.01289907 & 0.01289880 & 0.00213 \\
$\theta_{5}$ & 0.00179097 & 0.00179033 & 0.03540 \\
$\theta_{6}$ & 0.05717149 & 0.05717368 & 0.00384 \\
$\theta_{7}$ & 0.00013830 & 0.00013830 & 0.00306 \\
$\theta_{8}$ & 0.01255922 & 0.01255919 & 0.00023 \\
$\theta_{9}$ & 0.03510541 & 0.03510549 & 0.00022 \\
$\theta_{10}$ & 0.00048372 & 0.00048949 & 1.19333 \\
$\theta_{11}$ & 0.17268261 & 0.17267806 & 0.00263 \\
$\theta_{12}$ & 0.00954823 & 0.00954861 & 0.00403 \\
$\theta_{13}$ & 0.01056087 & 0.01056054 & 0.00309 \\
$\theta_{14}$ & 0.00020175 & 0.00020175 & 0.00243 \\
$\theta_{15}$ & 0.04329578 & 0.04329567 & 0.00026 \\
$\theta_{16}$ & 0.00130912 & 0.00130912 & 0.00029 \\
$\theta_{17}$ & 0.00146295 & 0.00146295 & 0.00003 \\
$\theta_{18}$ & 0.00037141 & 0.00037141 & 0.00072 \\
$\theta_{19}$ & 0.00003931 & 0.00003931 & 0.00030 \\
\hline
\end{tabular}

TABLE 4. Comparison between optimum and estimated sets of parameters when "noise-free" data $\left(d_{0}\right)$ are injected into the objective function.

They show that our algorithm is able to find again the optimum set of parameters used to simulate the noisefree data, with very high precision: the average and maximum relative errors between the 19 optimum and estimated parameters are about $0.07 \%$ and $1.2 \%$, respectively (expressed in absolute values). Furthermore, all model parameters, except $\theta_{10}$, are estimated with a precision lower than $0.05 \%$.

The evolution kinetics of model compartments simulated using estimated versus optimum parameter values are presented in Figure 12 (sampled compartments) and Figure 13 (non-sampled compartments). 


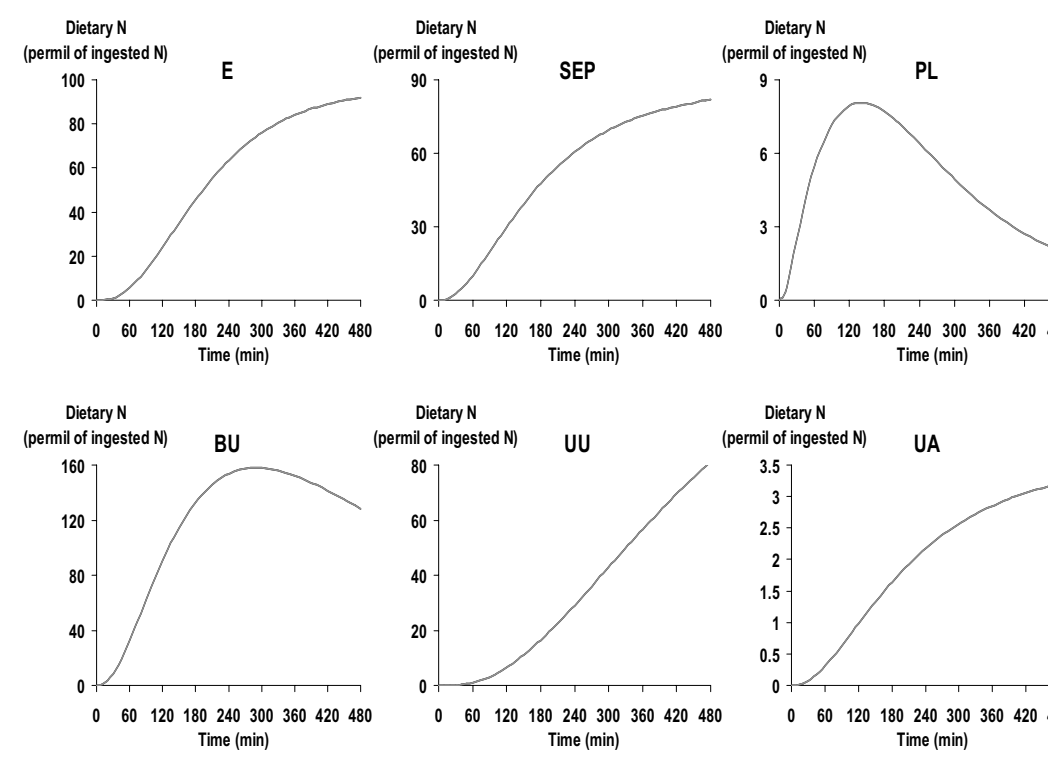

FiguRE 12. Predictions of the evolution kinetics for sampled compartments calculated using either the optimum set of parameters (black curve) or the estimated set of parameters (grey curve) when "noise-free" data $\left(d_{0}\right)$ are injected into the objective function.
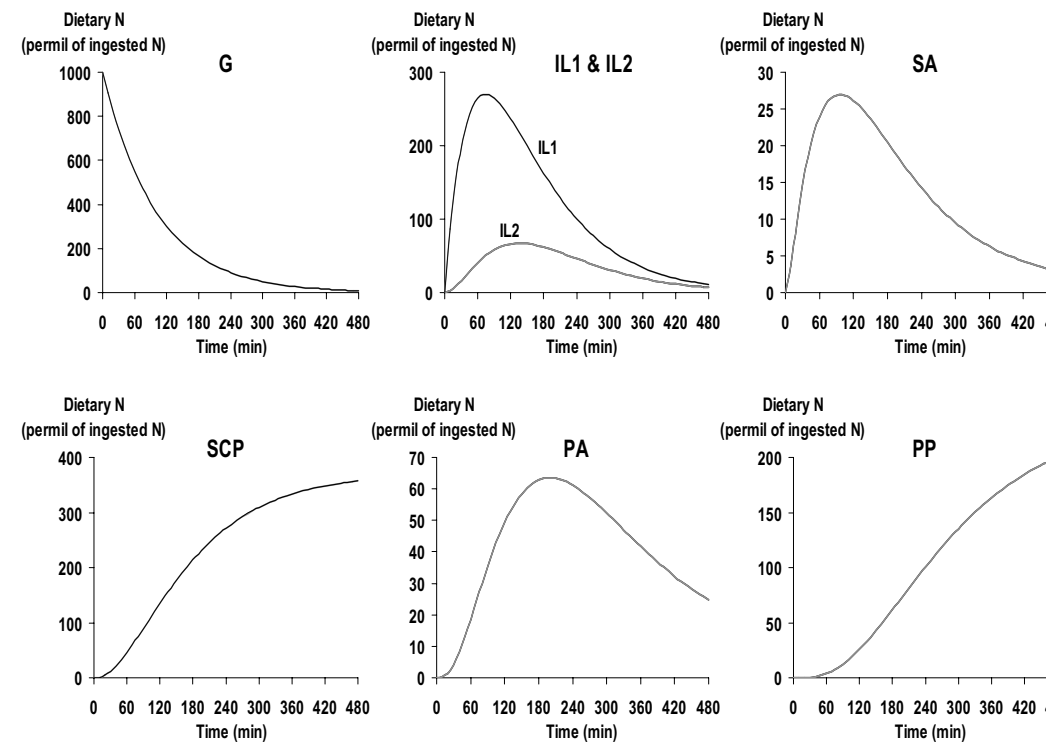

FiguRE 13. Predictions of the evolution kinetics for non-sampled compartments calculated using either the optimum set of parameters (black curve) or the estimated set of parameters (grey curve) when "noise-free" data $\left(d_{0}\right)$ are injected into the objective function. 
They show that the model predictions calculated using the estimated set of parameters are satisfactorily superimposed on those calculated with the optimum set of parameters, and this applies for both sampled and non-sampled compartments of the model. This means that, in the ideal context of noise-free data, our calibration method succeeds in estimating all model parameters with very high degree of precision, and thus in providing satisfactory predictions of the sizes of all model compartments.

\subsubsection{Noisy data $\left(d_{1}\right.$ and $\left.d_{2}\right)$}

The results of the calibration method when "low-noise" $\left(d_{1}\right)$ or "high-noise" $\left(d_{2}\right)$ data are injected into the objective function, are presented in Table 5.

\begin{tabular}{|c|c|c|c|c|c|}
\hline \multirow[b]{2}{*}{ Parameter } & \multirow[b]{2}{*}{$\begin{array}{l}\text { Optimum value } \\
\qquad\left(\min ^{-1}\right)\end{array}$} & \multicolumn{2}{|c|}{$d_{1}$} & \multicolumn{2}{|c|}{$d_{2}$} \\
\hline & & $\begin{array}{l}\text { Estimated value } \\
\qquad\left(\min ^{-1}\right)\end{array}$ & $\begin{array}{c}\text { Relative Error } \\
(\%)\end{array}$ & $\begin{array}{l}\text { Estimated value } \\
\qquad\left(\min ^{-1}\right)\end{array}$ & $\begin{array}{c}\text { Relative Error } \\
\qquad(\%)\end{array}$ \\
\hline$\theta_{1}$ & 0.01000170 & 0.01003368 & 0.32 & 0.00954457 & 4.57 \\
\hline$\theta_{2}$ & 0.00545349 & 0.00546366 & 0.19 & 0.00555255 & 1.82 \\
\hline$\theta_{3}$ & 0.01232039 & 0.01214851 & 1.40 & 0.01267226 & 2.86 \\
\hline$\theta_{4}$ & 0.01289907 & 0.01281316 & 0.67 & 0.01318219 & 2.19 \\
\hline$\theta_{5}$ & 0.00179097 & 0.00120250 & 32.86 & 0.00240708 & 34.40 \\
\hline$\theta_{6}$ & 0.05717149 & 0.05814667 & 1.71 & 0.06270203 & 9.67 \\
\hline$\theta_{7}$ & 0.00013830 & 0.00013250 & 4.19 & 0.00012229 & 11.57 \\
\hline$\theta_{8}$ & 0.01255922 & 0.01272895 & 1.35 & 0.01365536 & 8.72 \\
\hline$\theta_{9}$ & 0.03510541 & 0.03525330 & 0.42 & 0.03586055 & 2.15 \\
\hline$\theta_{10}$ & 0.00048372 & 0.00025500 & 47.28 & 0.00025500 & 47.28 \\
\hline$\theta_{11}$ & 0.17268261 & 0.16929332 & 1.96 & 0.15755942 & 8.76 \\
\hline$\theta_{12}$ & 0.00954823 & 0.00907060 & 5.00 & 0.00840858 & 11.94 \\
\hline$\theta_{13}$ & 0.01056087 & 0.00992134 & 6.06 & 0.00994375 & 5.84 \\
\hline$\theta_{14}$ & 0.00020175 & 0.00012025 & 40.40 & 0.00018558 & 8.01 \\
\hline$\theta_{15}$ & 0.04329578 & 0.04383942 & 1.26 & 0.04633971 & 7.03 \\
\hline$\theta_{16}$ & 0.00130912 & 0.00130375 & 0.41 & 0.00117112 & 10.54 \\
\hline$\theta_{17}$ & 0.00146295 & 0.00146455 & 0.11 & 0.00147691 & 0.95 \\
\hline$\theta_{18}$ & 0.00037141 & 0.00037806 & 1.79 & 0.00039746 & 7.01 \\
\hline$\theta_{19}$ & 0.00003931 & 0.00003870 & 1.55 & 0.00004289 & 9.10 \\
\hline
\end{tabular}

TABLE 5. Comparison between optimum and estimated sets of parameters when "low-noise" $\left(d_{1}\right)$ and "high-noise" $\left(d_{2}\right)$ data are injected into the objective function.

They show that our algorithm is able to find again the optimum set of parameters used to simulate noisy data $d_{1}$ and $d_{2}$ with satisfactory precision. In fact, the average over the 19 coordinates of the relative error between the optimum and estimated sets of parameters is about $8 \%$ and $10 \%$ for $d_{1}$ and $d_{2}$, respectively (expressed in absolute values). Furthermore, with the exception of parameters $\theta_{5}, \theta_{7}, \theta_{10}$ and $\theta_{14}$, all model parameters are estimated with very satisfactory precision, the average over the 15 remaining coordinates of the relative error between optimum and estimated values being inferior to $2 \%$ and $6 \%$ for $d_{1}$ and $d_{2}$, respectively. In fact, we could not hope that our calibration method would estimate the model parameters with better precision than the experimental error introduced in the data injected into the objective function. The experimental error introduced in data $d_{1}$ and $d_{2}$ being supposed to follow a gaussian distribution of standard deviations $\sigma_{i j}=\frac{1}{100} y_{j}\left(i \Delta t, \theta^{\star}\right)$ and $\sigma_{i j}=\frac{\mathbf{5}}{100} y_{j}\left(i \Delta t, \theta^{\star}\right)$, respectively (using the notations defined in section 1.2.2), this 
implies in particular that $95 \%$ of noisy data $z_{j}\left(t_{i}\right)$ should fall within 2 standard deviations of the mean, that is the predicted value $y_{j}\left(i \Delta t, \theta^{\star}\right)$. We therefore consider it satisfactory that most of the relative errors between the optimum and estimated parameters are found to be of the order of $2 \times \mathbf{1}=2 \%$ and $2 \times \mathbf{5}=10 \%$, respectively. The cases of parameters $\theta_{5}, \theta_{7}, \theta_{10}$ and $\theta_{14}$ are particular. As previously shown in section 1.3 .3 , these parameters have almost no influence on the sampled compartments, and thus on the objective function. This can also be seen in Figure 14, with respect to parameter $\theta_{7}$ for instance: the representation of the objective function in three dimensions ( $\theta_{6}$ and $\theta_{7}$ varying, the other parameters being set at their optimum values) indicates that small changes in $\theta_{7}$ will never lead to significant variations in the objective function value. The objective function surface is similar for parameters $\theta_{5}, \theta_{10}$ and $\theta_{14}$. This may explain the difficulties encountered when trying to estimate these parameters with satisfactory precision.

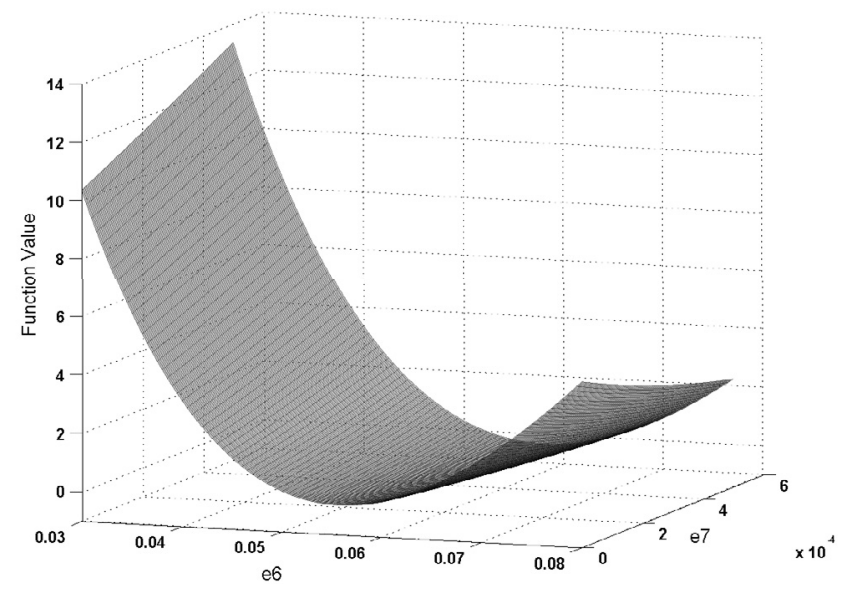

Figure 14. Plot of the objective function in case of "noise-free" data in two directions $\left(e_{6}, e_{7}\right)$.

The evolution kinetics of model compartments are presented in Figure 15 (sampled compartments) and Figure 16 (non-sampled compartments) when "low-noise" data $\left(d_{1}\right)$ are injected into the objective function, and in Figure 17 (sampled compartments) and Figure 18 (non-sampled compartments) when "high-noise" data $\left(d_{2}\right)$ are injected into the objective function.

Figures 15 and 17 show that the model predictions for the sampled compartments calculated using the estimated set of parameters are satisfactorily superimposed on those calculated with the optimum set of parameters, whatever the "experimental" data injected into the objective function $\left(d_{1}\right.$ or $\left.d_{2}\right)$. The results are also very satisfactory regarding the non-sampled compartments, when "low-noise" data $\left(d_{1}\right)$ are injected into the objective function (Figure 16). In the case of "high-noise" data $\left(d_{2}\right)$, two compartments, SA and PP, seem to be more sensitive to noise than the others (Figure 18). In fact, the relative error between the optimum and estimated sizes of compartment SA at the peak of the curve is about 9\% (expressed in absolute value), whereas the relative error between the optimum and estimated sizes of compartment PP at the last sampling point is about $8 \%$ (expressed in absolute value). Once again, errors in model predictions are found to be of the order of the "experimental" error introduced in data $d_{2}$. Compartments SA and PP are the most affected compartments in the model, mainly because SA is a central compartment that depends on parameters with the strongest influence on the system and PP is at the end of a catenary structure and, is therefore affected by all the errors accumulated upstream. 

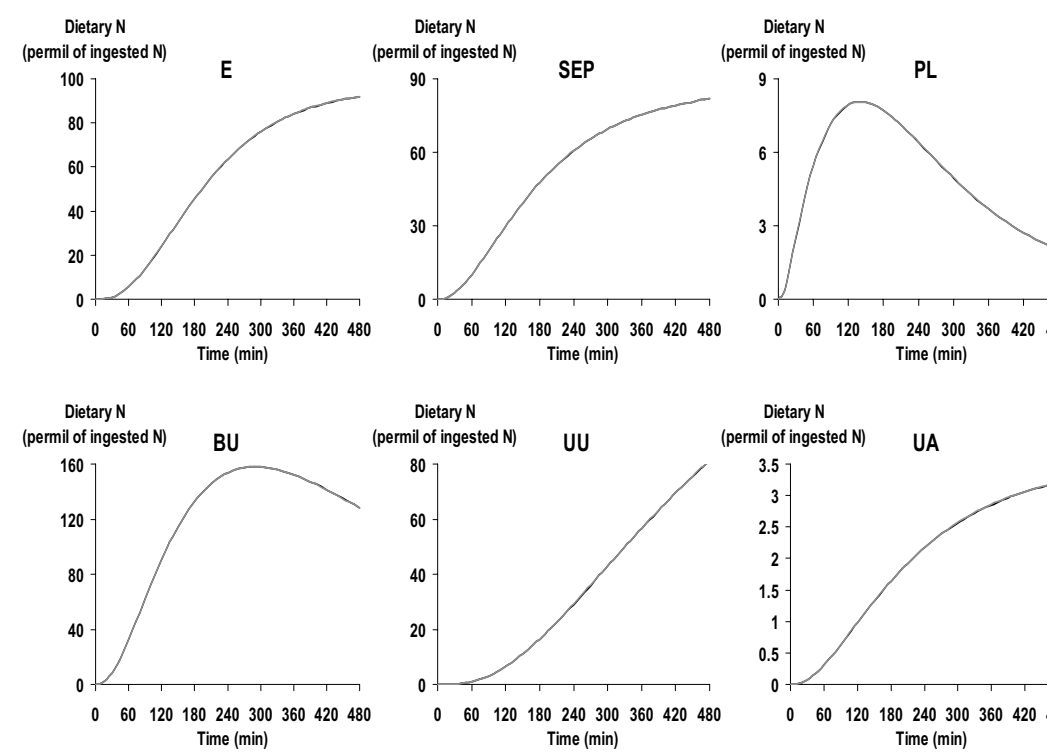

Figure 15. Predictions of the evolution kinetics for sampled compartments calculated using either the optimum set of parameters (black curve) or the estimated set of parameters (grey curve) when "low-noise" data $\left(d_{1}\right)$ are injected into the objective function.
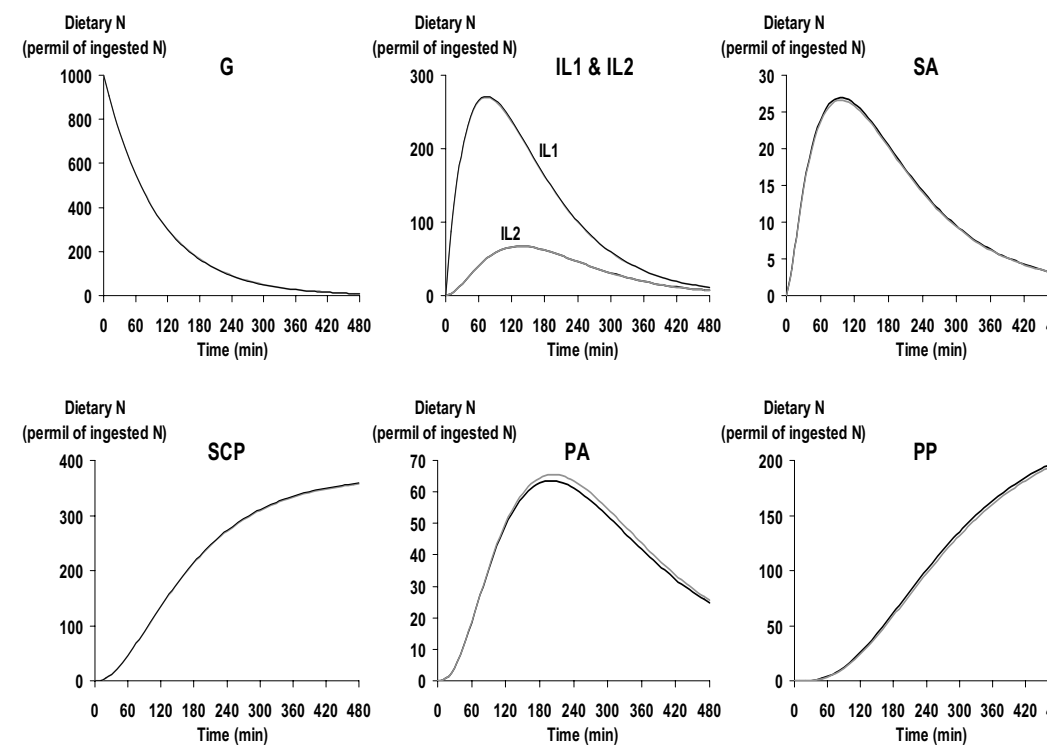

FIGURE 16. Predictions of the evolution kinetics for non-sampled compartments calculated using either the optimum set of parameters (black curve) or the estimated set of parameters (grey curve) when "low-noise" data $\left(d_{1}\right)$ are injected into the objective function. 

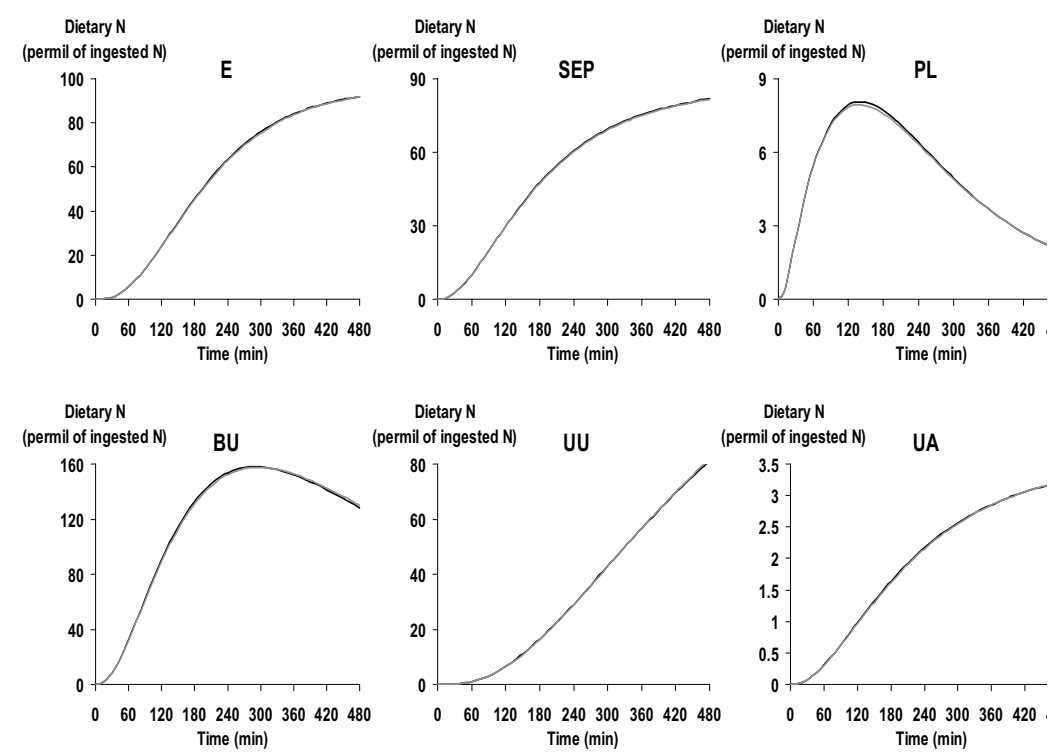

FIGURE 17. Predictions of the evolution kinetics for sampled compartments calculated using either the optimum set of parameters (black curve) or the estimated set of parameters (grey curve) when "high-noise" data $\left(d_{2}\right)$ are injected into the objective function.
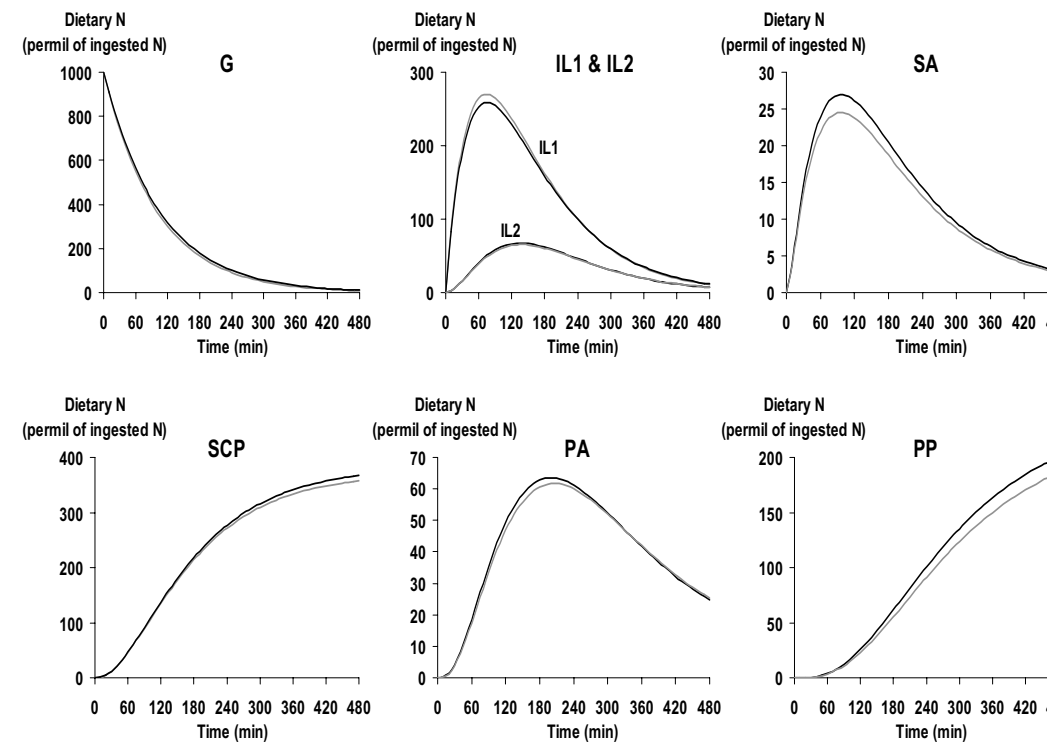

FIGURE 18. Predictions of the evolution kinetics for non-sampled compartments calculated using either the optimum set of parameters (black curve) or the estimated set of parameters (grey curve) when "high-noise" data $\left(d_{2}\right)$ are injected into the objective function. 


\section{Conclusion}

This paper introduces and validates a parameter estimation method that enables proper calibration of the metabolic model proposed by Fouillet et al. [18]. During this study, we worked either in the ideal context of noise-free data, or within a more realistic framework of noisy data, the noise component being known and controlled. The next step to validate our calibration method will thus be to test its behavior in the case of experimental data obtained from clinical experiments and associated with unknown noise. It will then probably be necessary to refine the error model used in this study, since it is well known that the success of a parameter estimation process is strongly dependent on the weight chosen for each sample point [13,27]. Considerable efforts should therefore be made to determine the sample variances at the different experimental points. In our experimental context, it is not possible to deduce experimentally (even roughly) the variations in error variances as a function of sampling times and sampled compartments, since we can not produce numerous replicates of our samples from the same individual for both ethical and financial reasons. To overcome this limitation, two main strategies can be adopted. The first consists in reducing the level of noise contained in the experimental data as much as possible, firstly during the clinical experiment and secondly during compartmental analysis (by smoothing the data before injecting them into the objective function). The second strategy consists in postulating a general error model characterized by a certain number of parameters that should be estimated alongside the model parameters during the calibration process. This latter strategy is more satisfactory from a theoretical point of view, but the first is easier to implement because the number of model parameters to be estimated is already quite large. By injecting smoothed data into the objective function, one could reduce the error made on estimated values of the model parameters, and therefore on the evolution kinetics of model compartments. Although our calibration method still needs to be perfected, it represents an important step towards the development of a dynamic model enabling the simulation of postprandial dietary $\mathrm{N}$ distribution in humans. Such a model constitutes a predictive and non-invasive tool that could highlight the acute postprandial mechanisms occurring in tissues and regulating the dynamic transfer of dietary $\mathrm{N}$ between organs. It may provide us with a deeper insight into the cascade of transient and dynamic metabolic processes involved in controlling dietary $\mathrm{N}$ distribution throughout the body, which plays an important role in replenishing protein stores during the postprandial phase. It may also enable determination of the organ-specific valorization of dietary protein in the non-steady state in humans, allowing further definition of the notion of protein quality. Moreover, it may in particular provide an opportunity to compare dietary $\mathrm{N}$ regional valorization and metabolism under different conditions (nature and quantity of proteins, lipids, carbohydrates and other nutrients in the meal, etc.), in order to optimize food composition as a function of physiological and nutritional status. Such a model will thus provide valuable insight into the dynamics and adaptative features of protein metabolism in humans.

The authors would like to thank E. Cancès and J.F. Gerbeau for organizing CEMRACS 2004, where stimulating discussions between mathematicians and biologists enabled us to make significant progress towards understanding and resolving our mathematical problem. We aknowledge in particular E. Cancès, J.F. Delmas, A. Gloria and G. Turinici from the École Nationale des Ponts et Chaussées (Paris, France), E. Oudet from the Université de Savoie (Le Bourget du lac, France), A. Saidi from the Institut de Recherche Mathématique Avancée (Strasbourg, France) and P. Michel from the École Nationale Supérieure (Paris, France).

\section{REFERENCES}

[1] Anderson D.H., Compartmental modeling and tracer kinetics, Lecture notes in biomathematics, vol. 50, Springer-Verlag, Berlin (1983).

[2] Anderson R.M. and May R.M., Infectious diseases of humans, Oxford: Oxford University Press (1991).

[3] Bard Y., Nonlinear parameter estimation, Academic Press, N.Y. (1974).

[4] Barton R.R. and Ivey J.S., Nelder-Mead simplex modifications for simplex optimization, Management Science 42 (1996): $954-$ 973.

[5] Biolo G., Tessari P., Inchiostro S., Bruttomesso D., Fongher C., Sabadin L., Fratton M.G., Valerio A. and Tiengo A., Leucine and phenylalanine kinetics during mixed meal ingestion: a multiple tracer approach, Am J Physiol 262 (1992): E455-63. 
[6] Bos C., Mahe S., Gaudichon C., Benamouzig R., Gausseres N., Luengo C., Ferriere F., Rautureau J. and Tome D., Assessment of net postprandial protein utilization of [15N]-labelled milk nitrogen in humans, Br J Nutr 81 (1999): 221-226.

[7] Carraro F., Rosenblatt J. and Wolfe R.R., Isotopic determination of fibronectin synthesis in humans. Metabolism 40 (1991): $553-561$.

[8] Carson Y. and Cobelli C., Modeling methodology for physiology and medicine, Academic Press, San Diego, CA, USA (2001).

[9] Carson Y. and Maria A., Simulation optimization: methods and applications, Proceedings of the 1997 Winter Simulation Conference.

[10] Castillo L., Chapman T.E., Yu Y.M., Ajami A., Burke J.F. and Young V.R., Dietary arginine uptake by the splanchnic region in adult humans, Am J Physiol 265 (1993): E532-9.

[11] Cobelli C., Saccomani M.P., Tessari P., Biolo G., Luzi L. and Matthews D.E., Compartmental model of leucine kinetics in humans, Am J Physiol Endocrinol Metab 261 (1991): E539-E550.

[12] Cobelli C., Carson E.R., Finkelstein L. and Leaning M.S., Validation of simple and complex models in physiology and medicine, Am J Physiol Regulatory Integrative Comp Physiol 246 (1984): R259-R266.

[13] Cobelli C. and Caumo A., Using what is accessible to measure that which is not: necessity of model of system, Met 47 (1998): 1009-1035.

[14] Cobelli C. and Foster D.M., Compartmental models: theory and practice using the SAAMII software system, Math Model Exp Nutr (1998): 79-101.

[15] DiStephano J.J. and Landaw E.M., Multiexponential, multicompartimental, and noncompartimental modeling. I. Methodological limitations and physiological interpretations, Am J Physiol 246 (1984): R651-664.

[16] Esposito W.R. and Floudas C.A., Global optimization in parameter estimation of nonlinear algebraic models via the error-invariables approach. Ind Eng Chem Res 37 (1998): 1841-1858.

[17] Fouillet H., Gaudichon C., Mariotti F., Mahe S., Lescoat P., Huneau J.F. and Tome D., Compartmental modeling of postprandial dietary nitrogen distribution in humans, Am J Physiol Endocrinol Metab 279 (2000): E161-E175.

[18] Fouillet H., Gaudichon C., Bos C., Mariotti F. and Tome D., Contribution of plasma proteins to splanchnic and total anabolic utilization of dietary nitrogen in humans, Am J Physiol Endocrinol Metab 279 (2003): E88-97.

[19] Finsterle S. and Najita J., Robust estimation of hydrogeologic model parameters, Water Resour Res 34 (1998): $2939-2947$.

[20] Gaudichon C., Mahe S., Benamouzig R., Luengo C., Fouillet H., Dare S., Van Oycke M., Ferriere F., Rautureau J. and Tome D., Net postprandial protein utilization of [15N]-labelled milk protein nitrogen is influenced by diet composition in humans, J Nutr 124 (1999).

[21] Gausseres N., Mahe S., Benamouzig R., Luengo C., Ferriere F., Rautureau J. and Tome D., [15N]-labeled pea flour protein nitrogen exhibits good ileal digestibility and postprandial retention in humans, J Nutr 127 (1997): 1160-1165.

[22] Green M.H. and Green J.B., The application of compartmental analysis to research in nutrition, Annu Rev Nutr 10 (1990): 41-61.

[23] Green M.H., Introduction to modeling, J Nutr 122 (1992): 690-694.

[24] Hoerr R.A., Matthews D.E., Bier D.M. and Young V.R., Effects of protein restriction and acute refeeding on leucine and lysine kinetics in young men, Am J Physiol 264 (1993): E567-75.

[25] Humphrey D.G. and Wilson J.R., A revised simplex search procedure for stochastic simulation response surface optimization, Proceedings of the 1998 Winter Simulation Conference, (1998): 751-759.

[26] Humphrey D.G. and Wilson J.R., A revised simplex search procedure for stochastic simulation response surface optimization, Informs J Computing, Vol. 12 (2000), no 4: 272-283.

[27] Jacquez J.A., Compartmental analysis in biology and medicine (3 ed), BioMedware, Ann Arbor, MI, USA (1996).

[28] Kendall M.G. and Stuart A., The advanced theory of statistics, Vol. 2, Charles Griffin and Co., Ltd, London (1961).

[29] Lagarias J.C., Reeds J.A., Wright M.H. and Wright P.E., Convergence properties of the Nelder-Mead simplex method in low dimensions, SIAM J Optim, Vol. 9 (1998), no. 1: 112-147.

[30] Lewis R.M., Torczon V. and Trosset M.W., Direct search methods: then and now, NASA-CR-2000-210125, ICASE Report no. 2000-26.

[31] Mendes P. and Kell D.B., Non-linear optimization of biochemical pathways: applications to metabolic engineering and parameter estimation, BioInformatics, Vol.14 (1998), no. 14: 869-883.

[32] Miller J.C. and Miller J.N., Statistics for Analytical Chemistry, Ellis Horwood Series in Analytical Chemistry, ed. R.A. Chalmers and M. Masson, Chichester (1984): 56.

[33] Millward D.J., Fereday A., Gibson N.R. and Pacy P.J., Human adult amino acid requirements: [1-13C]leucine balance evaluation of the efficiency of utilization and apparent requirements for wheat protein and lysine compared with those for milk protein in healthy adults, Am J Clin Nutr 72 (2000): 112-21.

[34] Moles C.G., Mendes P. and Banga J.R., Parameter estimation in biochemical pathways: a comparison of global optimization methods, Genome Research 13 (2003): 2467-2474.

[35] Nelder J.A. and Mead R., A simplex method for function minimization, Computer J 7 (1965): $308-313$.

[36] SAS/STAT 6.03, SAS Institute, Cary, NC.

[37] Volpi E., Mittendorfer B., Wolf S.E. and Wolfe R.R., Oral amino acids stimulate muscle protein anabolism in the elderly despite higher first-pass splanchnic extraction, Am J Physiol 277 (1999): E513-E520. 
[38] Waterlow J.C., 15N end-product methods for the study of whole body protein turnover, Proc Nutr Soc 40 (1981): 317-320.

[39] Wilks S.S., Mathematical Statistics, John Wiley and Sons, N.Y. (1962).

[40] Wolfe R.R., Radioactive and stable isotope tracers in biomedicine, New York, NY: Wiley-Liss (1992).

[41] Wutzke K., Heine W., Drescher U., Richter I. and Plath C., 15N-labelled yeast protein - a valid tracer for calculating wholebody protein parameters in infants: a comparison between [15N]-yeast protein and [15N]-glycine. Hum Nutr Clin Nutr 37 (1983): 317-327.

[42] Yen J, Liao J.C., Lee B. and Randolph D., A Hybrid Approach to Modeling Metabolic System Using Genetic Algorithm and Simplex Method, to appear in IEEE Transactions on Systems, Man, and Cybernetics (1995). 